\title{
Does a Single Eigenstate Encode the Full Hamiltonian?
}

\author{
James R. Garrison ${ }^{1,2}$ and Tarun Grover ${ }^{3,4}$ \\ ${ }^{1}$ Department of Physics, University of California, Santa Barbara, California 93106, USA \\ ${ }^{2}$ Joint Quantum Institute and Joint Center for Quantum Information and Computer Science, \\ National Institute of Standards and Technology and University of Maryland, \\ College Park, Maryland 20742, USA \\ ${ }^{3}$ Kavli Institute for Theoretical Physics, University of California, Santa Barbara, California 93106, USA \\ ${ }^{4}$ Department of Physics, University of California at San Diego, La Jolla, California 92093, USA
}

(Received 31 August 2017; revised manuscript received 14 December 2017; published 30 April 2018)

\begin{abstract}
The eigenstate thermalization hypothesis (ETH) posits that the reduced density matrix for a subsystem corresponding to an excited eigenstate is "thermal." Here we expound on this hypothesis by asking: For which class of operators, local or nonlocal, is ETH satisfied? We show that this question is directly related to a seemingly unrelated question: Is the Hamiltonian of a system encoded within a single eigenstate? We formulate a strong form of ETH where, in the thermodynamic limit, the reduced density matrix of a subsystem corresponding to a pure, finite energy density eigenstate asymptotically becomes equal to the thermal reduced density matrix, as long as the subsystem size is much less than the total system size, irrespective of how large the subsystem is compared to any intrinsic length scale of the system. This allows one to access the properties of the underlying Hamiltonian at arbitrary energy densities (or temperatures) using just a single eigenstate. We provide support for our conjecture by performing an exact diagonalization study of a nonintegrable 1D quantum lattice model with only energy conservation. In addition, we examine the case in which the subsystem size is a finite fraction of the total system size, and we find that, even in this case, many operators continue to match their canonical expectation values, at least approximately. In particular, the von Neumann entanglement entropy equals the thermal entropy as long as the subsystem is less than half the total system. Our results are consistent with the possibility that a single eigenstate correctly predicts the expectation values of all operators with support on less than half the total system, as long as one uses a microcanonical ensemble with vanishing energy width for comparison. We also study, both analytically and numerically, a particle-number conserving model at infinite temperature that substantiates our conjectures.
\end{abstract}

DOI: 10.1103/PhysRevX.8.021026

\section{INTRODUCTION}

Given a local Hamiltonian, what information about the system is encoded in a single eigenstate? If the eigenstate happens to be a ground state of the Hamiltonian, a tremendous amount of progress can be made on this question for Lorentz invariant systems [1,2], especially conformal field theories (CFTs) [3-6], and for topological phases [7-9]. For example, one can read off the central charge of a CFT from the ground state entanglement [3-5], while for topological phases, essentially all "topological data" such as braiding statistics of anyons can be extracted from the degenerate ground states [8-10]. In this paper, we argue that a single finite energy density eigenstate of an

Published by the American Physical Society under the terms of the Creative Commons Attribution 3.0 License. Further distribution of this work must maintain attribution to the author $(s)$ and the published article's title, journal citation, and DOI.
Subject Areas: Condensed Matter Physics, Quantum Physics, Statistical Physics ergodic quantum many-body Hamiltonian is sufficient to determine the properties of the system at all temperatures.

It is not very surprising that the ground states of quantum many-body systems contain some information about their excitations. This is because an entanglement cut often mimics an actual physical cut through the system, thus exposing the underlying excitations along the entangling boundary [9]. The same intuition is tied to the fact that the ground state entanglement satisfies a "boundary law" of entanglement entropy [11,12]; that is, the von Neumann entanglement entropy $S_{1}=-\operatorname{tr}_{A}\left(\rho_{A} \log \left(\rho_{A}\right)\right)$ of the ground state corresponding to a subsystem $A$ scales with the size of the boundary of subsystem $A$.

How does the nature of information encoded evolve as one goes from the ground state to an excited eigenstate? Typically, there always exist eigenstates with energy $E$ just above the ground state that continue to satisfy an area law of entanglement. These are the eigenstates that have zero energy density, i.e., $\lim _{V \rightarrow \infty}\left[\left(E-E_{0}\right) / V\right]=0$, where $E_{0}$ is the ground state energy and $V$ is the total volume of the 
system. These eigenstates can often be interpreted as the action of a sum of local operators acting on the ground state; for example, in a system with spontaneous symmetry breaking, one can construct an eigenstate consisting of a few magnons by a superposition of spin-flips acting on the ground state. Furthermore, the level spacing between two contiguous low-lying excitations scales as $\delta E \sim 1 / L^{\alpha}$, where $\alpha>0$ depends on dimensionality and the phase of matter under consideration. In this paper, we will instead be concerned with excited eigenstates that have a finite energy density, i.e., $\lim _{V \rightarrow \infty}\left[\left(E-E_{0}\right) / V\right] \neq 0$. For notational convenience, we will set $E_{0}=0$ for the remainder of this paper.

As argued by Srednicki [13], the question of how (and whether) an isolated quantum system eventually reaches thermal equilibrium is closely related to properties of its finite energy density eigenstates. Let us consider an arbitrary (nonequilibrium) initial state, with finite energy density and subextensive energy fluctuations, as might be prepared by performing a quantum quench [14]. Evolving such a state under the Hamiltonian $H$ for sufficient time is expected to lead to the predictions dictated by the basic tenets of equilibrium statistical mechanics, if the system thermalizes. Such an expectation leads to the "eigenstate thermalization hypothesis" (ETH) $[13,15,16]$, which stipulates that the thermalization occurs at the level of each individual eigenstate. An alternative approach by Deutsch [15], which is based on perturbing an integrable system by a small integrability breaking term, leads to the same suggestion. If ETH holds true, then, in the thermodynamic limit, the equal-time correlators of an operator with respect to a finite energy density eigenstate $|\psi\rangle$ are precisely equal to those derived from the canonical ensemble, i.e.,

$$
\langle\psi|O| \psi\rangle=\frac{\operatorname{tr}\left(O e^{-\beta H}\right)}{\operatorname{tr}\left(e^{-\beta H}\right)},
$$

where $\beta$ is chosen such that Eq. (1) holds true when $O=H$, the Hamiltonian. Henceforth, we will use the notation $|\psi\rangle_{\beta}$ to denote an eigenstate whose energy density corresponds to temperature $\beta^{-1}$. A notable exception to ETH is a manybody localized system in the context of strongly disordered interacting quantum systems [17-23], which fails to thermalize and does not satisfy Eq. (1). The possibility [24-30], or impossibility [31-34], of the violation of ETH without disorder has also been discussed recently.

In this paper, we restrict ourselves to systems where ETH, as defined by Eq. (1), holds. However, Eq. (1) alone is incomplete unless one also specifies the class of operators for which it holds. For example, one well-known nonlocal operator for which Eq. (1) breaks down is the projection operator $|\psi\rangle\langle\psi|$ onto the eigenstate $|\psi\rangle$ that enters Eq. (1); the left-hand side of Eq. (1) yields unity for this operator, while the right-hand side is exponentially small in the volume, a clear disagreement. On that note, it is often mentioned that in systems where Eq. (1) does hold, it does so only for "few-body" operators [35-37], where, to our knowledge, the precise meaning of a few-body operator has not been clarified (see Ref. [38] for related discussion). In this paper, we conjecture and provide numerical evidence that Eq. (1) holds for all operators within a subsystem $A$ when the volume $V_{A}$ of subsystem $A$ satisfies $V_{A} \ll V$ (or, more precisely, when $V_{A} / V \rightarrow 0$ as $V \rightarrow \infty$ ). We also explore the validity of ETH when the subsystem fraction $V_{A} / V$ is taken to be finite as $V_{A}, V \rightarrow \infty$. While we find in this case that Eq. (1) fails to hold for some operators, our results are consistent with the possibility that ETH holds for all operators, as long as a microcanonical ensemble is used for comparison. [We will return to this case shortly, after first considering the implications of Eq. (1).]

The satisfaction of Eq. (1) for all operators in a subsystem $A$ is equivalent to the statement that the reduced density matrix $\rho_{A}\left(|\psi\rangle_{\beta}\right)=\operatorname{tr}_{\bar{A}}|\psi\rangle_{\beta \beta}\langle\psi|$ corresponding to an eigenstate $|\psi\rangle_{\beta}$ is given by [23]

$$
\rho_{A}\left(|\psi\rangle_{\beta}\right)=\rho_{A, \text { canonical }}(\beta),
$$

where

$$
\rho_{A, \text { canonical }}(\beta)=\frac{\operatorname{tr}_{\bar{A}}\left(e^{-\beta H}\right)}{\operatorname{tr}\left(e^{-\beta H}\right)},
$$

$\bar{A}$ being the complement of $A$. Note that the trace in the denominator is over the whole Hilbert space. The equality in Eq. (2a) means that the density matrices become elementwise equal in any basis as $V \rightarrow \infty$.

One immediate consequence of Eq. (2a) is that the thermodynamical properties of a system at arbitrary temperatures can be calculated using a single eigenstate. For example, Eq. (2a) implies that to the leading order, the Renyi entropies $S_{\alpha}\left(=-[1 /(\alpha-1)] \log \left[\operatorname{tr}_{A}\left(\rho_{A}^{\alpha}\right)\right]\right)$ for an eigenstate $|\psi\rangle_{\beta}$ corresponding to a subsystem $A$ with $V_{A} \ll V$ are given by $[39,40]$

$$
S_{\alpha}=\frac{\alpha}{\alpha-1} V_{A} \beta(f(\alpha \beta)-f(\beta)),
$$

where $f(\beta)$ is the free energy density at temperature $\beta^{-1}$. The above equation allows one to access the free energy density $f$ at an arbitrary temperature by varying $\alpha$. Note that Eq. (3) holds only to the leading order because Renyi entropies $S_{\alpha}$ receive additional subleading contributions due to the conical singularity induced at the boundary of subsystem $A$ [3-5]. In the limit $\alpha \rightarrow 1$, one recovers the equality between the von Neumann entanglement entropy $S_{1}$ and the thermal entropy $S_{\text {th }}=V_{A} s_{\text {th }}(\beta)$, where $s_{\text {th }}(\beta)$ is the thermal entropy density at temperature $\beta^{-1}$, a result which was argued to hold in Ref. [41] for the special case of two weakly coupled ergodic systems. We emphasize that these results could not be derived from Eq. (1) alone if it were 
to hold only for local operators, since entanglement entropies do not correspond to the expectation value of any local operator. We also note that Refs. [40,42] simulated the thermal Renyi entropy $S_{\alpha}$ [starting with the expression on the right-hand side of Eq. (2a)] using quantum Monte Carlo to access the properties of the system at temperature $(\alpha \beta)^{-1}$. Of course, quantum Monte Carlo methods are not well suited to verifying ETH since they cannot access properties of a single eigenstate [the left-hand side of Eq. (2a)].

We will also discuss an approximate, but more intuitive form of ETH, given by

$$
\rho_{A}\left(|\psi\rangle_{\beta}\right) \approx \frac{e^{-\beta H_{A}}}{\operatorname{tr}_{A}\left(e^{-\beta H_{A}}\right)},
$$

where $H_{A}$ is the projection of the original Hamiltonian onto subsystem $A$. This form is approximate compared to Eq. (2a) because, generically, it does not capture the correlations near the boundary correctly because of the somewhat arbitrary truncation scheme used to obtain $H_{A}$. Nevertheless, Eqs. (2a) and (2b) both yield the same results for all bulk quantities, such as the Renyi entropy densities, as well as correlation functions of operators that have support only far from the boundary. Equation (2b) is also related to the notion of "canonical typicality" [43-45], which states that, when $V_{A} \ll V$, the vast majority of pure states within a microcanonical energy shell will reproduce the canonical ensemble within subregion $A$, assuming the interaction between $A$ and $\bar{A}$ to be small. Thus, when Eq. (2b) holds, it implies that finite energy density eigenstates are locally equivalent to typical states.

A central task of this paper is to check the validity of Eqs. (2a) and (2b) and their consequences for model nonintegrable systems. As already mentioned, we will argue that ETH allows one to calculate thermodynamical quantities, as well as correlators, at all energy densities (or temperatures) using only a single eigenstate. We will demonstrate this explicitly by studying a quantum 1D model numerically.

We also explore the validity of ETH in the more general case, where subsystem $A$ spans a finite fraction $f \equiv$ $V_{A} / V>0$ of the total system size. In this case, the thermodynamic limit $V_{A}, V \rightarrow \infty$ is taken in a way that holds $f$ constant. Although Eq. (1) does not hold for all operators in this case, we find preliminary evidence that it nevertheless holds for many operators when $f<1 / 2$. In particular, our results strongly indicate that $f<1 / 2$ is sufficient to guarantee equivalence between the von Neumann entropy density of a pure eigenstate and the thermal entropy density at the corresponding temperature. This is in contrast to Ref. [46], where it was argued that such an equivalence holds only in the limit $f^{*} \rightarrow 0$. Recently [47,48], the requirement $f^{*} \rightarrow 0$ was substantiated using analytical and large scale numerical calculations for free fermions, an integrable system. Our results indicate that the $f^{*} \rightarrow 0$ requirement is likely a consequence of the integrable nature of the models in Refs. [47,48].
On the other hand, we are able to construct operators for which Eq. (1) fails when $V_{A} / V$ is nonzero. These operators include powers of the Hamiltonian $H_{A}$ restricted to subsystem $A$, as well as certain "nonequithermal" operators (which we define below) when the subsystem fraction is greater than a critical $O(1)$ number, $f^{*}$. Crucially, these cases each correspond to operators for which the canonical and microcanonical ensembles predict different results (they are only strictly equivalent when $V_{A} \ll V$ ).

In fact, the results of Refs. [49,50] suggest that a single eigenstate is expected to reproduce the results of a microcanonical ensemble, rather than the canonical ensemble, when $0<f<1 / 2$. A microcanonical ensemble with energy width $\Delta E$ is given by an equal-weighted mixture of nearby eigenstates,

$$
\rho_{\mathrm{mc}}(E)=\frac{1}{\mathcal{N}} \sum_{E^{\prime} \in[E-\Delta E / 2, E+\Delta E / 2]}|\psi\rangle_{E^{\prime} E^{\prime}}\langle\psi|,
$$

where $|\psi\rangle_{E^{\prime}}$ denotes an eigenstate of energy $E^{\prime}$. For the purposes of this paper, the energy window $\Delta E$ is chosen to vanish as the thermodynamic limit is taken in a way such that the number of states $\mathcal{N}$ in the energy window $[E-\Delta E / 2, E+\Delta E / 2]$ is always large. We thus introduce a third version of ETH that we explore in this paper, given by

$$
{ }_{E}\langle\psi|O| \psi\rangle_{E}=\operatorname{tr}\left[O \rho_{\mathrm{mc}}(E)\right] .
$$

If this equation holds for all operators $O$ within subregion $A$, it implies the equivalence

$$
\rho_{A}\left(|\psi\rangle_{E}\right)=\rho_{A, \mathrm{mc}}(E)
$$

where $\rho_{A, \mathrm{mc}}(E) \equiv \operatorname{tr}_{\bar{A}}\left[\rho_{\mathrm{mc}}(E)\right]$ is the microcanonical ensemble restricted to subregion $A$.

As we will discuss, our results are consistent with the possibility that Eq. (4) holds for all operators that span less than half the system, which is equivalent to the notion that Eq. (2c) holds precisely in the thermodynamic limit as long as $f<1 / 2$. Thus, ETH may hold for all operators with support on less than half the total system, as long as a microcanonical ensemble with vanishing energy width is used for comparison.

The paper is organized as follows. Section II discusses general considerations for the validity of ETH and introduces a division of all operators spanning a macroscopic subregion into two distinct classes, which have different requirements for ETH to hold. Section III illustrates some general features of ETH by studying properties of a hardcore boson model with global particle-number conservation for infinite temperature eigenstates. Section IV introduces the model that we study in the remainder of the paper, the transverse field Ising model with longitudinal field. Section V focuses on the entanglement entropies at 
finite temperature. Section VI studies the validity of ETH when $V_{A} \ll V$ by providing a close look into the entanglement Hamiltonian, focusing on its spectrum and Schmidt vectors. This section also demonstrates the validity of Eq. (2a) when $V_{A} \ll V$ by considering the trace norm distance between both sides. Section VII explores the validity of ETH when $V_{A} / V$ is taken to be finite as $V \rightarrow \infty$. Section VIII provides an application, by using the reduced density matrix from a single eigenstate to predict correlators and free energy densities at all (finite) temperatures. Section IX summarizes our results and provides thoughts for future discussion.

\section{GENERAL CONSIDERATIONS}

\section{A. Determining the Hamiltonian from microstates in classical statistical mechanics}

Suppose that, for an isolated system described by classical statistical mechanics in a total volume $V$, we are given access to all classical microstates in a small energy window $[E, E+\Delta E]$, where $\Delta E \sim \sqrt{V}$ is on the order of the energy fluctuations in the total system if it were coupled to a thermal bath, and, thus, all microstates correspond to the same energy density. We pose the question: Does this information suffice to determine the underlying Hamiltonian, assuming that the Hamiltonian is local? The answer is indeed yes, following the standard procedure of obtaining the canonical ensemble from a microcanonical ensemble. In particular, let us make a fictitious division of the system into $A$ and $\bar{A}$, such that $V_{A} \ll V_{\bar{A}}$, and count the number of times a particular configuration $C_{A}$ appears in subsystem $A$. This determines the probability distribution for finding a given configuration, $P\left(C_{A}\right)$. If all microstates are equally likely, then [51]

$$
P\left(C_{A}\right)=\frac{e^{-\beta E\left(C_{A}\right)}}{\sum_{\left\{C_{A}\right\}} e^{-\beta E\left(C_{A}\right)}},
$$

where $E\left(C_{A}\right)$ is the energy in subsystem $A$. One may now invert this equation to obtain the energy $E\left(C_{A}\right)=$ $-(1 / \beta) \log \left(P\left(C_{A}\right)\right)$, up to an irrelevant constant shift of energy. In a classical statistical mechanical system, $E\left(C_{A}\right)$ is the Hamiltonian for subsystem $A$. In particular, knowing $E\left(C_{A}\right)$, one may now calculate any thermodynamic property at any temperature. Here it is crucial to note that Eq. (5) does not assume that the energy density $E\left(C_{A}\right) / V_{A}$ equals the energy density $E / V$ of the microstates being sampled.

As discussed in the Introduction, we will provide evidence that the quantum mechanical analog of Eq. (5) is given by Eqs. (2a)-(2c). We now proceed to discuss the conditions under which Eqs. (2a)-(2c) are valid.

\section{B. Two classes of operators}

One of the central tasks of this paper is to investigate whether a single finite energy density eigenstate can contain information about a system at all temperatures. In terms of Eqs. (2a) and (2b), this is equivalent to asking whether a single eigenstate contains accurate information about microstates in subregion $A$ that have thermodynamically rare energy density. In this paper, we phrase this question in terms of which operators satisfy ETH. From this perspective, it is useful to separate operators into two classes with respect to a given Hamiltonian, $H$ :

Equithermal operators: When the full system is considered with respect to the canonical ensemble, the expectation value of an equithermal operator receives significant contribution only from microstates in subregion $A$ with thermodynamically common energy density (i.e., those corresponding to the temperature, $\beta^{-1}$ ). [Here, $A$ is the subregion spanned by the operator, and "microstates" in subregion $A$ are the eigenvectors of either $\rho_{A \text {,canonical }}(\beta)$ or $H_{A}$ (corresponding to Eq. (2a) or Eq. (2b), respectively).] Local operators fall into this class because there are no thermodynamically rare microstates on one site (or very few sites). This class also includes sums of local operators, as well as some nonlocal quantities, such as the energy variance and von Neumann entropy $\left(S_{1}\right)$ within some subregion. Essentially, equithermal operators are those for which the saddle point approximation is valid. Crucially, a single eigenstate of energy $E$ need not contain accurate information about energy densities away from $E / V$ for Eq. (1) to hold for equithermal operators.

Nonequithermal operators: Nonequithermal operators are those that, when considered with respect to the canonical ensemble, receive significant contribution from microstates with thermodynamically rare energy density, i.e., energy densities other than that corresponding to the temperature, $\beta^{-1}$. In many cases, these are operators specifically designed to probe thermodynamically rare microstates in a subregion of the system. For instance, an operator that includes a factor like $e^{-\beta^{\prime} H_{A}}$ is nonequithermal because it probes microstates of subregion $A$ at temperature $\left(\beta+\beta^{\prime}\right)^{-1}$. A projector onto the ground state (or any collection of energetically rare microstates) within a macroscopic subregion is also a nonequithermal operator. This class also includes all Renyi entropies $S_{\alpha}$ for $\alpha \neq$ 1 [39].

If handed an operator as a black box, determining whether it is most accurately characterized as equithermal or nonequithermal with respect to a Hamiltonian requires analyzing it on the subsystem spanned by the operator. Let $H_{A}$ be the Hamiltonian restricted to this subregion, and let $E_{n}^{(A)}$ and $\left|n^{(A)}\right\rangle$ denote the eigenvalues and eigenvectors, respectively, of $H_{A}$. Then, the expectation value $\langle O\rangle_{\beta, H_{A}}$ is equal to $\left(1 / Z_{A}\right) \sum_{n} e^{-\beta E_{n}^{(A)}}\left\langle n^{(A)}|O| n^{(A)}\right\rangle$, where $Z_{A}=\operatorname{tr}\left(e^{-\beta H_{A}}\right)$. Hence, we can create a weighted histogram of contributions 
$e^{-\beta E_{n}^{(A)}}\left|\left\langle n^{(A)}|O| n^{(A)}\right\rangle\right|$ with respect to energy density $E_{n}^{(A)} / V_{A}$. If, for various $\beta$, this histogram is peaked at the same energy density as the histogram for $e^{-\beta E_{n}^{(A)}}$ alone, then the operator is equithermal.

It is important to recognize that the concept of a nonequithermal operator becomes better defined as an operator spans a larger (more macroscopic) subregion of the system. However, even on subregions of moderate size (e.g., three or more sites), we can directly probe whether all microstates match between a single eigenstate and a thermal ensemble. If ETH [in the sense of Eq. (1) or Eq. (4)] is valid even for nonequithermal operators, it implies that a single eigenstate contains information about a system at all temperatures.

\section{ETH: Equithermal vs. nonequithermal operators}

Let us first consider the relationship between Eq. (1) and Eqs. (2a) and (2b). Equation (1) may be rewritten as

$$
\operatorname{tr}_{A}\left(\rho_{A} O\right)=\frac{\operatorname{tr}_{A}\left(O \operatorname{tr}_{\bar{A}}\left(e^{-\beta H}\right)\right)}{\operatorname{tr}\left(e^{-\beta H}\right)} .
$$

If this equation holds for all operators in a subsystem $A$, Hermitian as well as non-Hermitian, then one obtains Eq. (2a), $\rho_{A}\left(|\psi\rangle_{\beta}\right)=\rho_{A \text {,canonical }}(\beta)$. This is because one may expand both $\rho_{A}$ and $\rho_{A \text {,canonical }}$ in terms of the complete set of operators in subsystem $A$ and, by choosing appropriate $O$, prove that they are equal to each other element by element. One of the most important consequences of this equality is that it allows one to extract properties of the Hamiltonian at arbitrary temperatures using a single eigenstate, which is one of the central points of this paper.

We will now discuss ETH for both equithermal and nonequithermal operators. For each class of operator, we consider separately two cases: (i) when $V_{A} \ll V$ and (ii) when the ratio $f \equiv V_{A} / V$ is taken to be fixed and finite as $V_{A}, V \rightarrow \infty$.

\section{ETH for equithermal operators}

Let us first briefly discuss ETH for equithermal operators when $V_{A} \ll V$. The simplest case to consider is when $V_{A}$ is held fixed as $V \rightarrow \infty$. This generalizes the traditional definition of ETH to go beyond "few-body" operators and include those that span an arbitrary portion of the system, greater than any intrinsic length scale. The condition $V_{A} \ll V$ also includes the case when the limits $V_{A}$, $V \rightarrow \infty$ are taken such that $V_{A} / V \rightarrow 0$. If Eq. (2a) holds for fixed subsystem size, it is reasonable to expect that it holds in this case as well.

Let us now consider the validity of ETH for equithermal operators in the fixed-ratio limit, where $0<f<\frac{1}{2}$ is finite. In contrast to classical statistical mechanics, we expect that quantum mechanically, one does not require the constraint $V_{A} \ll V_{\bar{A}}$ for ETH to hold for a large class of equithermal operators. Indeed, as discussed below, several known results already point to the conclusion that ETH holds for at least some equithermal operators, as long as $V_{A}<V_{\bar{A}}$ with both $V_{A}, V_{\bar{A}} \rightarrow \infty$.

One piece of evidence that suggests that ETH might hold for equithermal operators as long as $V_{A}<V_{\bar{A}}$ comes from the study of quantum quenches in conformal field theories (CFTs). As shown in Ref. [52], the timedependent reduced density matrix $\rho_{A}(t)$ of a system initially prepared in a low-entanglement state, and evolved with a CFT Hamiltonian, approaches the canonical reduced density matrix, as long as $V_{A}<V / 2$, with $V_{A}$, $V \rightarrow \infty$. The author of Ref. [52] characterized the closeness between $\rho_{A}(t)$ and the thermal density matrix $\rho_{A, \text { canonical }}$ [Eq. (2a)] in terms of the operator overlap $I(t)=\operatorname{tr}\left(\rho_{A}(t) \rho_{A \text {,canonical }}\right) /\left[\operatorname{tr}\left(\rho_{A}^{2}(t)\right) \operatorname{tr}\left(\rho_{A, \text { canonical }}^{2}\right)\right]^{1 / 2}$, which is exponentially close to unity for $V_{A} / 2<t<V_{\bar{A}} / 2$. It is important to note that, in the thermodynamic limit, $I$ only receives contribution from eigenstates at temperature $\beta^{-1}$, so this only guarantees that equithermal operators will satisfy Eq. (1). It is also important to recognize that the initial states considered in Ref. [52] are constructed from applying an exponential of the Hamiltonian to the CFT boundary state. The initial states, therefore, have some knowledge of the Hamiltonian, which perhaps explains why the states relax to the canonical ensemble rather than a different, diagonal ensemble.

Another piece of evidence comes from the recent studies of large central charge conformal field theories [53-55]. In particular, the authors of Refs. [53,55] studied the entanglement entropy of pure eigenstates in finite temperature conformal field theories with large central charge. In the limit $V_{A}, V \gg 1 / T$, while keeping $V_{A} / V$ fixed, it was found that the entanglement entropy becomes equal to the thermal entropy at all nonzero temperatures as long as $V_{A}<V_{\bar{A}}$.

Lastly, the entanglement entropy for a random pure state is given by [56-58]

$$
S=-\log \left(\left|\mathcal{H}_{A}\right|^{-1}+\left|\mathcal{H}_{\bar{A}}\right|^{-1}-|\mathcal{H}|^{-1}\right),
$$

where $\left|\mathcal{H}_{A}\right|,\left|\mathcal{H}_{\bar{A}}\right|$, and $|\mathcal{H}|$ are the sizes of the Hilbert spaces of subsystems $A, \bar{A}$, and the total system $(=A \cup \bar{A})$, respectively. Thus, as soon as $V_{A}<V_{\bar{A}}$, one obtains $S=-\log \left(\left|\mathcal{H}_{A}\right|\right)$, which is indeed the thermal entropy for subsystem $A$ at infinite temperature. Since random pure states mimic eigenstates at infinite temperature (i.e., $\left.|\psi\rangle_{\beta=0}\right)$, this again suggests that the condition $V_{A}<V_{\bar{A}}$ is perhaps sufficient, at least for some operators.

On the other hand, there is a well-known equithermal operator for which Eq. (1) fails when the ratio of the subsystem to the total system size $f=V_{A} / V$ is finite [59]. When $f$ is finite, the energy variance of the reduced density matrix $\rho_{A}\left(|\psi\rangle_{\beta}\right)$ will be suppressed by a factor of $(1-f)$ compared with the value that the variance would have taken in the canonical ensemble. Ultimately, this is due to the fact that a single eigenstate has precisely zero energy variance 
$\left\langle(H-\langle H\rangle)^{2}\right\rangle$ in the full system, unlike in the canonical ensemble, where the variance scales proportionally with system size. This relationship can be expressed as

$$
\operatorname{tr}\left[\rho_{A}\left(|\psi\rangle_{\beta}\right) \mathcal{O}_{A, \beta}\right]=\frac{V_{\bar{A}}}{V} \operatorname{tr}\left[\rho_{A, \text { canonical }}(\beta) \mathcal{O}_{A, \beta}\right],
$$

where $\mathcal{O}_{A, \beta}=\left(H_{A}-\left\langle H_{A}\right\rangle_{\beta}\right)^{2}$ is the energy variance operator. We will explore implications of the subsystem energy variance mismatch between a single eigenstate and the canonical ensemble more carefully in Sec. VII A.

Of course, it is important to note that a suitably defined microcanonical ensemble has zero energy variance in the thermodynamic limit, just like a single eigenstate. This provides further evidence that a microcanonical ensemble is the appropriate ensemble to compare a single eigenstate with when $V_{A} / V$ is nonzero $[49,50]$ and, therefore, that Eq. (2c) is the most appropriate form of ETH to consider in this case. We will consider this equation's validity in Sec. VII B.

There is another issue to consider regarding the subsystem energy variance. For the case of time-evolved states, the full system energy variance is independent of time. For a given initial state, this variance may indeed be different from the energy variance expected in the canonical ensemble, which implies that the energy variance for any subsystem that is a finite fraction of the total system will disagree, even at long times [60]. In this case, the system will relax to a subsystem energy variance given by the diagonal ensemble, which depends upon the energy histogram of the initial state.

In summary, while we expect that Eq. (1) is obeyed by many equithermal operators when $V_{A} / V$ is nonzero, it cannot be satisfied by all such operators, since the subsystem energy variance provides an important counterexample. Instead, a more appropriate definition of ETH in this case is the one given by Eq. (2c), which compares the reduced density matrix from a single eigenstate to a microcanonical ensemble with vanishing energy window.

\section{ETH for nonequithermal operators}

The extra ingredient introduced by nonequithermal operators is that if ETH holds for them, then taking such an operator's expectation value with respect to a state $|\psi\rangle_{\beta}$ allows one to access the properties of the Hamiltonian at a temperature different than $\beta^{-1}$. For example, the Renyi entropy $S_{\alpha}$ corresponding to $\rho_{A}\left(|\psi\rangle_{\beta}\right)$ satisfies $S_{\alpha}=$ $[\alpha /(\alpha-1)] V_{A} \beta(f(\alpha \beta)-f(\beta))$, thus allowing one to access the free energy density at temperature $(\alpha \beta)^{-1}$.

Let us first consider the validity of ETH for nonequithermal operators when $V_{A} \ll V$. Remarkably, the results presented in the remainder of this paper demonstrate that ETH is valid for all nonequithermal operators in this limit. Thus, a single eigenstate of finite energy density contains knowledge of the properties of the system at all temperatures.

Now let us turn to the case in which $V_{A} / V$ is nonzero, which turns out to be much more subtle. First, we consider the potential validity of Eqs. (1) and (2a) in this case, which, for the purposes of the following discussion, we refer to as "canonical ETH." As mentioned in the previous subsection, there is already an equithermal operator for which this equation fails in this limit, namely, the subsystem energy variance. Thus, we do not expect that Eq. (1) will hold for all nonequithermal operators either when $f$ is nonzero. In addition, for a given ratio $V_{A} / V$ with both $V_{A}$, $V \rightarrow \infty$, there is a physical constraint on the range of energy densities for which the spectrum of $|\psi\rangle_{\beta}$, in principle, can match that of $\rho_{A \text {,canonical }}(\beta)$. To appreciate this, let us consider a slightly different problem-an arbitrary Hamiltonian of hardcore bosons with particlenumber conservation, at infinite temperature. We will consider an explicit example of such a system in the next section. Since the total particle-number operator $\hat{N}$ commutes with the Hamiltonian and satisfies the equation $\hat{N}=\hat{N}_{A}+\hat{N}_{\bar{A}}$, the reduced density matrix $\rho_{A}$ for a wave function $|\psi\rangle_{\beta=0}$ is block diagonal in the number of particles $N_{A}$ in subsystem $A$. Furthermore, if canonical ETH holds [as given by a generalization of Eqs. (2a) and (2b)], then the Schmidt decomposition is given by

$$
|\psi\rangle_{\beta=0}=\sum_{N_{A}=0}^{N} \sqrt{\lambda_{N_{A}}} \sum_{i}\left|u_{i}\right\rangle_{N_{A}} \otimes\left|v_{i}\right\rangle_{N-N_{A}},
$$

where $\lambda_{N_{A}}$ are the Schmidt coefficients in the sector $N_{A}$, and $\left|u_{i}\right\rangle_{N_{A}},\left|v_{i}\right\rangle_{N-N_{A}}$ are the corresponding eigenvectors. The label $i$ captures fluctuations of particles within a fixed sector $N_{A}$. Note that there is no index $i$ on $\lambda_{N_{A}}$ because we are at infinite temperature and all Schmidt states within a sector $N_{A}$ are equally likely.

The decomposition in Eq. (9) allows one to calculate properties of subsystem $A$ at infinite temperature even away from filling $N / V$, since the reduced density matrix $\rho_{A}$ will contain sectors with various densities $N_{A} / V_{A}$. However, there is both an upper limit and a lower limit on the density in subsystem $A$, since

$$
\max \left[N-\left(V-V_{A}\right), 0\right] \leq N_{A} \leq \min \left[N, V_{A}\right],
$$

and, thus, the particle density $N_{A} / V_{A}$ in subsystem $A$ satisfies

$$
\max [1-(1-n) / f, 0] \leq \frac{N_{A}}{V_{A}} \leq \min [n / f, 1],
$$

where $n \equiv N / V$ is the overall particle density and $f \equiv V_{A} / V$. A necessary condition for the wave function in Eq. (9) to encode properties of the system at all fillings is 


$$
f \leq \min [n, 1-n] .
$$

The above discussion, with some modifications, carries to systems with (only) energy conservation, at an arbitrary temperature. The Schmidt decomposition of an eigenstate $|\psi\rangle_{\beta}$ with eigenvalue $E$ may now be written as

$$
|\psi\rangle_{\beta}=\sum_{i} \sqrt{\lambda_{i}}\left|u_{i}\right\rangle \otimes\left|v_{i}\right\rangle
$$

The physical content of canonical ETH, as approximated in Eq. (2b), is that $\lambda_{i} \propto e^{-\beta E_{A, i}}$, where $E_{A, i}$ is the $i$ th energy eigenvalue of $H_{A}$ (the projection of the Hamiltonian to subsystem $A$ ), while $\left|u_{i}\right\rangle$ is the corresponding eigenstate of $H_{A}$. Following the discussion of particle number above, one might naively expect that $\left\langle u_{i}\left|H_{A}\right| u_{i}\right\rangle \leq E \forall\left|u_{i}\right\rangle$, since the energy density in subsystem $\bar{A}$ cannot be less than zero (which would correspond to region $\bar{A}$ being in its ground state). However, this argument has a loophole since, in contrast to the particle-number operator $\hat{N}$, the total Hamiltonian is not separable into subsystems $A$ and $\bar{A}$ : $H=H_{A}+H_{\bar{A}}+H_{A \bar{A}}$, which actually allows $\left\langle u_{i}\left|H_{A}\right| u_{i}\right\rangle$ to exceed $E$, as we will see in Sec. VII in the context of the model Hamiltonian in Eq. (30) below. To understand the constraint on $\left\langle u_{i}\left|H_{A}\right| u_{i}\right\rangle$ precisely, let us derive an expression that encapsulates the classical notion that the sum of energies in subsystem $A$ and $\bar{A}$ equals $E$.

We first note

$$
\begin{aligned}
\left\langle u_{i 0}\right| \otimes\left\langle v_{i 0}|H| \psi\right\rangle_{\beta} & =E\left\langle u_{i 0}\right| \otimes\left\langle v_{i 0} \mid \psi\right\rangle_{\beta} \\
& =E \sqrt{\lambda_{i 0}} .
\end{aligned}
$$

The above expression can be reevaluated using the decomposition $H=H_{A}+H_{\bar{A}}+H_{A \bar{A}}$ :

$$
\begin{aligned}
\left\langle u_{i 0}\right| \otimes\left\langle v_{i 0}|H| \psi\right\rangle_{\beta} \\
=\left\langle u_{i 0}\right| \otimes\left\langle v_{i 0}\left|H_{A}+H_{\bar{A}}+H_{A \bar{A}}\right| \psi\right\rangle_{\beta} \\
=\sqrt{\lambda_{i 0}}\left\langle u_{i 0}\left|H_{A}\right| u_{i 0}\right\rangle+\sqrt{\lambda_{i 0}}\left\langle v_{i 0}\left|H_{\bar{A}}\right| v_{i 0}\right\rangle \\
\quad+\sum_{j} \sqrt{\lambda_{j}}\left\langle u_{i 0}\left|\otimes\left\langle v_{i 0}\left|H_{A \bar{A}}\right| u_{j}\right\rangle \otimes\right| v_{j}\right\rangle .
\end{aligned}
$$

Equating the two ways to calculate the same expression, one finds

$$
\begin{aligned}
& \left\langle v_{i 0}\left|H_{\bar{A}}\right| v_{i 0}\right\rangle+\sum_{j} \sqrt{\frac{\lambda_{j}}{\lambda_{i 0}}}\left\langle u_{i 0}\left|\otimes\left\langle v_{i 0}\left|H_{A \bar{A}}\right| u_{j}\right\rangle \otimes\right| v_{j}\right\rangle \\
& =E-\left\langle u_{i 0}\left|H_{A}\right| u_{i 0}\right\rangle .
\end{aligned}
$$

Because of the variational principle, the energy density in subregion $\bar{A}$ cannot be less than that of the ground state. Allowing negative energy contributions due to boundary terms in $H_{\bar{A}}$ gives the inequality $\left\langle v_{i 0}\left|H_{\bar{A}}\right| v_{i 0}\right\rangle \geq-c L^{d-1}$, where $c$ is a constant (recall that, in our convention, the ground state energy for the full Hamiltonian is set to zero). Since both $E$ and $\left\langle u_{i 0}\left|H_{A}\right| u_{i 0}\right\rangle$ scale as $L^{d}$, the only way that $\left\langle u_{i 0}\left|H_{A}\right| u_{i 0}\right\rangle$ can exceed $E$ is if the second term on the left-hand side of Eq. (17), viz. $E_{\text {boundary }} \stackrel{\text { def }}{=} \sum_{j} \sqrt{\lambda_{j} / \lambda_{i 0}}\left\langle u_{i 0}\left|\otimes\left\langle v_{i 0}\left|H_{A \bar{A}}\right| u_{j}\right\rangle \otimes\right| v_{j}\right\rangle$, is negative and scales as $L^{d}$. When that happens, canonical ETH no longer holds, as we now argue on general grounds, and as we will also demonstrate numerically for a lattice Hamiltonian in Sec. VII. To see this, we reiterate that canonical ETH requires that (i) $\left|u_{i}\right\rangle$ 's are approximate eigenstates of $H_{A}$, and (ii) $\lambda_{i} \propto e^{-\beta\left\langle u_{i}\left|H_{A}\right| u_{i}\right\rangle}=e^{-\beta E_{A, i}}$. Firstly, when $\left\langle u_{i 0}\left|H_{A}\right| u_{i 0}\right\rangle<E$ so that canonical ETH could in principle hold, the $E_{\text {boundary }}$ term can be neglected because the "diagonal term" in $E_{\text {boundary }}$ (i.e., the term corresponding to $j=i 0$ ) scales as the boundary $\left(\propto L^{d-1}\right)$ and is thus subleading, while the off-diagonal terms scale as $e^{-L^{d}}$ and thus vanish in the thermodynamic limit (recall that $\left.V_{\bar{A}}>V_{A}\right)$. On the other hand, when $\left\langle u_{i 0}\left|H_{A}\right| u_{i 0}\right\rangle>E$, the $\left|v_{i 0}\right\rangle$ 's now correspond to states of zero energy density, and the aforementioned argument for neglecting offdiagonal terms is no longer valid. So, let us assume that $\left\langle u_{i 0}\left|H_{A}\right| u_{i 0}\right\rangle>E$ and each $\left|u_{i 0}\right\rangle$ continues to be an eigenstate of $H_{A}$. Thus, one requires that

$$
\int d e^{\prime} \sqrt{\frac{\lambda\left(e^{\prime}\right)}{\lambda(e)}} M\left(e, e^{\prime}\right) e^{S\left(e^{\prime}\right)} \propto g(e) / L^{d-1},
$$

where we have taken the continuum limit, and where $\lambda(e)$ denotes the Schmidt eigenvalue corresponding to an eigenvector $|u\rangle$ at energy density $e$, while $M\left(e, e^{\prime}\right)=\left\langle u(e)\left|\otimes\left\langle v(e)\left|H_{A \bar{A}}\right| u\left(e^{\prime}\right)\right\rangle \otimes\right| v\left(e^{\prime}\right)\right\rangle$ and $g(e)=$ $e-\left\langle u(e)\left|H_{A}\right| u(e)\right\rangle / L^{d}$. It is obvious from Eq. (18) that $\lambda(e) \propto e^{-\beta E_{A}}=e^{-\beta e f L^{d}}$ is no longer the solution. In fact, the only way for the integral on the left-hand side of Eq. (18) not to have any exponential dependence on $L$ (as required by the right-hand side) is that the integrand itself does not have such dependence, i.e., $\sqrt{\lambda\left(e^{\prime}\right) / \lambda(e)} \propto e^{-S\left(e^{\prime}\right)} / M\left(e, e^{\prime}\right)$. This implies a breakdown of canonical ETH when $\left\langle u_{i 0}\left|H_{A}\right| u_{i 0}\right\rangle>E$.

The above discussion implies that for a given wave function and bipartition, the maximum energy density that is potentially accessible in a subsystem $A$, such that the corresponding Schmidt weight satisfies canonical ETH, is

$$
e^{*}=\min \left(E / V_{A}, e_{\max }\right)=\min \left(e / f, e_{\max }\right),
$$

where $e=E / V$ is the energy density corresponding to the wave function and $e_{\max }$ is the maximum energy density for the Hamiltonian $H$ (recall that $e_{\max }$ can be finite for latticeregularized quantum systems, e.g., for models of fermions or spins/hardcore bosons). Above, we have assumed that 
$e<e_{\max } / 2$. In the case when $e>e_{\max } / 2$, the range of available energies is instead bounded from below by $\max \left[0, e_{\max }(1-1 / f)-e / f\right]$. If our goal is to capture fluctuations in the system for all energy densities so that all nonequithermal operators can potentially satisfy canonical ETH, we obtain an analog of Eq. (12) for the energy: $E / V_{A} \geq e_{\max }$ and $\left(e_{\max } V-E\right) / V_{A} \geq e_{\max }$. Expressed in terms of the fraction $f=V_{A} / V$ and the energy density of the eigenstate $e=E / V$, this constraint is

$$
f \leq f^{*} \equiv \min \left[\frac{e}{e_{\max }}, 1-\frac{e}{e_{\max }}\right] .
$$

Let us emphasize that the above constraint is a necessary condition for canonical ETH to hold for all nonequithermal operators, not a sufficient one. Just as some equithermal operators cannot satisfy Eq. (1) when $f$ is finite, we expect that there also exist nonequithermal operators for which the same equation fails when $f$ is finite, even when the above condition holds. Even so, significant deviation in the eigenvalue spectrum begins where this constraint breaks down, as our numerical results will demonstrate in Sec. VII. We also emphasize that the above considerations apply only to canonical ETH as described by Eqs. (1), (2a), and (2b).

We now briefly consider the potential validity of Eqs. (4) and (2c) ("microcanonical ETH") when $V_{A} / V$ is held fixed as $V_{A}, V \rightarrow \infty$. To our knowledge, there is nothing conceptual that prevents the exact equivalence given by Eq. (4) when $V_{A} / V<\frac{1}{2}$; in other words, these general considerations leave open the possibility that microcanonical ETH holds for all operators that span less than half the system. This potentially includes nonequithermal operators; operators for which equivalence between canonical and microcanonical ensembles breaks down; and operators that cannot be represented as a sum of local operators (including, e.g., the von Neumann entanglement entropy $S_{A}=\left\langle-\log \rho_{A}\right\rangle$, which we study in Sec. V). Of course, it is clear that Eq. (2c) cannot be valid when $V_{A} / V>\frac{1}{2}$ because the Schmidt values of a single eigenstate are symmetric under the transformation $A \leftrightarrow \bar{A}$, while a microcanonical ensemble (mixture of many nearby eigenstates) does not have this property.

\section{Summary}

Let us summarize the discussion in this section.

1. We conjecture that ETH holds for all local and nonlocal equithermal operators as long as $V_{A} \ll V$. This implies that ETH is not restricted only to few-body operators (as can be seen in the limit $V_{A} / V \rightarrow 0$ as $\left.V_{A}, V \rightarrow \infty\right)$.

2. We conjecture that ETH also holds for all nonequithermal operators when $V_{A} \ll V$. It follows that a single eigenstate contains information about all energy densities available to the system.

3. Determining the full Hamiltonian from a single eigenstate is equivalent to the satisfaction of Eq. (1) for both equithermal and nonequithermal operators. Our results provide strong evidence that this is true when
$V_{A} \ll V$. Therefore, one should be able to extract information about the full Hamiltonian at arbitrary energy densities (or temperatures) using a single eigenstate. We demonstrate this explicitly in Sec. VIII.

We also considered, in this section, the case where the subregion spans a finite fraction $0<V_{A} / V<\frac{1}{2}$ of the system as $V_{A}, V \rightarrow \infty$. We demonstrated that there are both equithermal and nonequithermal operators for which the single eigenstate expectation value disagrees with that of the canonical ensemble, and, hence, Eq. (2a) must break down in this case. However, the considerations in this section leave open the possibility that ETH holds precisely when $V_{A} / V<\frac{1}{2}$ as long as a microcanonical ensemble with a vanishing energy window is used for comparison [Eq. (2c)]. We find further evidence supporting this possibility in Sec. VII.

\section{A WARMUP: EIGENSTATES AT INFINITE TEMPERATURE}

\section{A. Von Neumann and Renyi entropy}

By definition, the thermal entropy reaches a maximum at infinite temperature. Together with Eq. (35), this implies that when ETH holds, eigenstates at "infinite temperature" are ones where the entanglement entropy is at its maximum. Consider a 1D transverse field Ising model with longitudinal field, $H=\sum_{i=1}^{L}\left(\sigma_{i}^{z} \sigma_{i+1}^{z}+h_{x} \sigma_{i}^{x}+h_{z} \sigma_{i}^{z}\right)$. Here, the von Neumann entropy $S_{1}$ takes its maximum possible value when the eigenvalues of the reduced density matrix are all equivalent to one another. Thus, from counting the basis size of the reduced Hilbert space, we expect for infinite temperature eigenstates that each eigenvalue of the reduced density matrix will approach $2^{-L_{A}}$ in the thermodynamic limit when $f=L_{A} / L<\frac{1}{2}$. From this, it follows that the Renyi entropies at infinite temperature satisfy

$$
S_{\alpha}=L_{A} \log 2
$$

that is, they are independent of Renyi index $\alpha$. The left panel of Fig. 1 shows how the entropies $S_{1}$ through $S_{4}$ together match this predicted value at the infinite temperature point for a $L=21$ system with periodic boundary conditions and subsystem size $L_{A}=4$. In general, as $L \rightarrow \infty$, the $T=\infty$ entropy density is given by $S_{\alpha} / L_{A}=\log 2$.

Now let us instead consider a model with an additional conservation law, namely, particle-number conservation. Consider a 1D chain of hardcore bosons,

$$
\begin{aligned}
H= & -\sum_{i}\left(t b_{i}^{\dagger} b_{i+1}+t^{\prime} b_{i}^{\dagger} b_{i+2}+\text { H.c. }\right) \\
& +\sum_{i}\left(V n_{i} n_{i+1}+V^{\prime} n_{i} n_{i+2}\right),
\end{aligned}
$$

where $n_{i} \equiv b_{i}^{\dagger} b_{i}$. We focus on this system with periodic boundary conditions at the nonintegrable point $t=V=1$ and $t^{\prime}=V^{\prime}=0.96$. This model was previously studied and shown to exhibit ETH in Refs. [61,62]. 

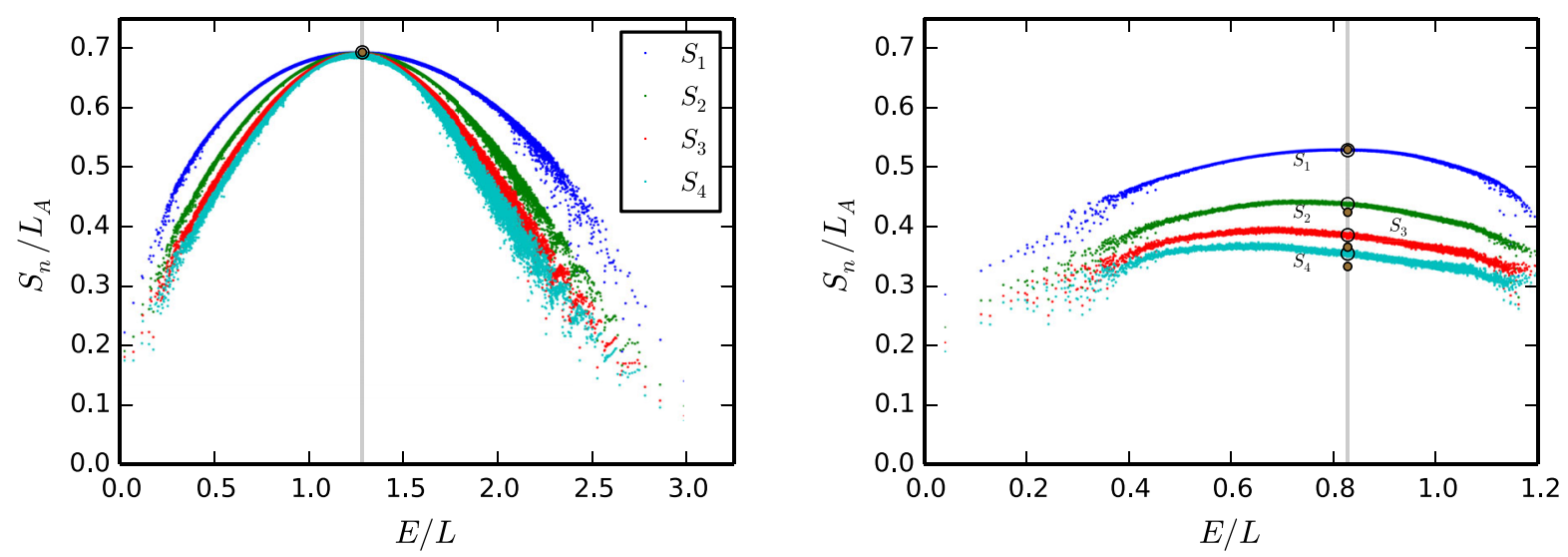

FIG. 1. Entanglement entropies $S_{1}$ through $S_{4}$ for a model with no conservation law [left panel, given by Eq. (30) at $L=21$ ] and a model with particle-number conservation [right panel, given by Eq. (22) at $L=27$, with filling $N=6$ ]. We use the parameters mentioned in the text to place each model at a nonintegrable point. In each case, we consider eigenstates in the $k=1$ sector, with subsystem size $L_{A}=4$. The grey vertical line denotes infinite temperature (point of maximum $S_{1}$ ), and the black circles mark the theoretical predictions for the entanglement entropies there. The brown markers denote the theoretical values of the entropies in the limit $L_{A}, L \rightarrow \infty$ while $L_{A} / L \rightarrow 0$, as given by Eqs. (26) and (27).

Because of particle-number conservation, the reduced density matrix from any pure state is block diagonal, with each block corresponding to some filling number $N_{A}$ of the subsystem $A$. The block of the reduced density matrix $\rho_{A}^{\left(N_{A}\right)}$ corresponding to filling $N_{A}$ is a $d_{N_{A}} \times d_{N_{A}}$ matrix, where $d_{N_{A}} \equiv\left(\begin{array}{c}L_{A} \\ N_{A}\end{array}\right)$. At infinite temperature and for $L_{A} / L<\frac{1}{2}$, the eigenvalues of $\rho_{A}$ must be equal to one another within a given block, but the eigenvalues in different blocks will be different: They are proportional to $\left(\begin{array}{l}L-L_{A} \\ N-N_{A}\end{array}\right)$, the number of microstates consistent with such a configuration in subsystem $A$. Taking into account that $\operatorname{tr}\left(\rho_{A}\right)=1$, one finds that each of the $d_{N_{A}} \equiv\left(\begin{array}{c}L_{A} \\ N_{A}\end{array}\right)$ eigenvalues of $\rho_{A}^{\left(N_{A}\right)}$ is given by $\lambda_{N_{A}} \equiv\left(\begin{array}{c}L-L_{A} \\ N-N_{A}\end{array}\right) /\left(\begin{array}{c}L \\ N\end{array}\right)$. The spectrum of $\rho_{A}$ that we find for a single eigenstate (as shown in Fig. 2) is in agreement

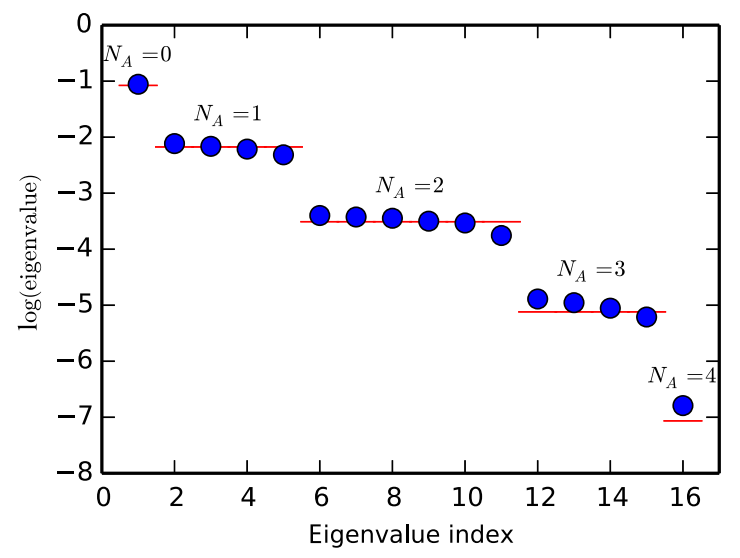

FIG. 2. Eigenvalue spectrum of the reduced density matrix of an infinite temperature eigenstate, $\rho_{A}\left(|\psi\rangle_{\beta=0}\right)$, for the hardcore boson model Eq. (22), with $L=27, L_{A}=4$, and filling $N=6$. The red lines plot the theoretical value of each eigenvalue in the thermodynamic limit, determined from the filling $N_{A}$ of the sector in which it lies. with that of the thermal reduced density matrix $\rho_{A \text {,canonical }}(\beta=0)$ studied in Ref. [40], consistent with ETH.

With this, the von Neumann entropy at infinite temperature becomes

$$
S_{1}=-\sum_{N_{A}} d_{N_{A}} \lambda_{N_{A}} \log \lambda_{N_{A}}
$$

and the Renyi entropies are given by

$$
S_{\alpha}=-\frac{1}{\alpha-1} \log \left(\sum_{N_{A}} d_{N_{A}} \lambda_{N_{A}}^{\alpha}\right)
$$

where the sums over $N_{A}$ are restricted to subsystem particle fillings $N_{A}$ that satisfy the constraint in Eq. (10). The above expressions are valid when $L_{A} / L<\frac{1}{2}$.

Because the eigenvalues are nonuniform, the Renyi entropies $S_{\alpha}$ at infinite temperature depend on the Renyi index $\alpha$, in contrast to an energy-only conserving model. The right panel of Fig. 1 shows how the actual values of $S_{1}$ through $S_{4}$ match those predicted by the above counting argument. [Note that if we had instead studied this model at half filling (i.e., "zero chemical potential"), the Renyi entropy densities at infinite temperature would again be independent of $\alpha$, just like for the energy-only conserving model.]

For comparison, we also calculate $S_{\alpha}$ analytically in the thermodynamic limit. For simplicity, we consider the limits $L, N, L_{A} \rightarrow \infty$, such that $n=N / L$ is held constant, while $L_{A} / L \rightarrow 0$. In these limits, one can evaluate the expressions in Eq. (24) using Stirling's approximation $\log (x !) \approx$ $x \log (x)-x$. One finds that, in the limits considered, $S_{\alpha}$ receives contribution only from $N_{A}$ given by 


$$
N_{A}^{*}=\frac{L_{A}}{1+\left(\frac{1}{n}-1\right)^{\alpha}} .
$$

Thus, $S_{\alpha}$ probes the system at the filling $N_{A}^{*} / L_{A}=$ $1 /\left\{1+[(1 / n)-1]^{\alpha}\right\}$, which is different than the actual filling $n$, unless $\alpha=1$ (which corresponds to the von Neumann entanglement entropy). This also immediately leads to expressions for Renyi and von Neumann entanglement entropies in the thermodynamic limit:

$$
S_{\alpha} / L_{A}=-\frac{1}{\alpha-1} \log \left[n^{\alpha}+(1-n)^{\alpha}\right]
$$

and

$$
S_{1} / L_{A}=-[n \log (n)+(1-n) \log (1-n)] .
$$

We plot these values in Fig. 1 for comparison. Remarkably, even with the small system sizes we can access, the difference between the exact finite-size result (obtained by counting over all sectors) and the result valid in the thermodynamic limit is quite small.

In the above derivation, it is also possible to relax the restriction $L_{A} / L \rightarrow 0$ as $L_{A}, L \rightarrow \infty$. We then find that $N_{A}^{*}$ is given by the solution to

$$
N_{A}^{*}=\frac{L_{A}}{1+\left(\frac{1-f}{n-f N_{A}^{*} / L_{A}}-1\right)^{\alpha}},
$$

which reduces to Eq. (25) when $f \rightarrow 0$.

Let us note a few things about this equation:

(1) When $\alpha=1$, the solution is $N_{A}^{*}=n L_{A}$, regardless of $f$. Thus, the von Neumann entropy always probes the system at its given filling, even when $f$ is finite. Further analysis shows that Eq. (27) holds generally when $f<\frac{1}{2}$.

(2) When the system is at half filling $\left(n=\frac{1}{2}\right)$, the solution is $N_{A}^{*}=\frac{1}{2} L_{A}$, regardless of $f$ or $\alpha$.

(3) When $\alpha>1,0<f<\frac{1}{2}$, and $n \neq \frac{1}{2}$, the filling fraction $N_{A}^{*} / L_{A}$ probed by the Renyi entropy $S_{\alpha}$ actually depends on $f$. As a result, the Renyi entropies for a given $L_{A}$ depend on $f$. This can be contrasted with the von Neumann entropy, which is independent of $f$ as long as $f<\frac{1}{2}$. The right panel of Fig. 1 illustrates this nicely: the analytical $f \rightarrow 0$ prediction for the von Neumann entropy [Eq. (27)] matches the corresponding numerical result quite well, but the Renyi entropies differ significantly because $f=4 / 27$ is finite.

We expect that analogous features hold true also for the model that conserves only energy, which we will discuss in the later sections.

\section{B. Subsystem energy variance}

Let us also consider the average subsystem filling variance of the particle-number conserving system given by Eq. (22) at infinite temperature. Following the logic in the previous subsection, the subsystem occupancy is given by the hypergeometric distribution. While the average subsystem filling is $\left\langle N_{A}\right\rangle=n L_{A}=N f$, the variance in this quantity for a single eigenstate is

$$
\left\langle\left(N_{A}-\left\langle N_{A}\right\rangle\right)^{2}\right\rangle=L_{A}(1-f) n(1-n) \frac{L}{L-1} .
$$

Both the filling and its variance are proportional to $L_{A}$ as expected, but the variance includes an additional factor $(1-f)$, which causes it to be suppressed compared with the grand canonical ensemble when $f$ is finite. In Sec. VII A we will witness a similar suppression of the subsystem energy variance when the condition $L_{A} / L \rightarrow 0$ is relaxed.

\section{MODEL HAMILTONIAN WITH ONLY ENERGY CONSERVATION}

To develop some understanding of the questions posed in the Introduction, we study in detail throughout the remainder of the paper a finite $1 \mathrm{D}$ quantum spin-1/2 chain with the following Hamiltonian:

$$
H=\sum_{i=1}^{L}\left(\sigma_{i}^{z} \sigma_{i+1}^{z}+h_{x} \sigma_{i}^{x}+h_{z} \sigma_{i}^{z}\right) .
$$

We set $h_{x}=0.9045$ and $h_{z}=0.8090$ such that the model is far away from any integrable point and is thus expected to satisfy ETH in the sense of Eq. (1), as shown in Ref. [63]. We use periodic boundary conditions throughout.

We diagonalized the Hamiltonian in Eq. (30) for system sizes up to $L=21$, obtaining all eigenvalues and eigenstates. As we hinted earlier, we assigned to each eigenstate a temperature $\beta^{-1}$ by finding the value $\beta$ for which the energy expectation value in the canonical ensemble matches the energy of the eigenstate:

$$
\frac{\langle\psi|H| \psi\rangle}{\langle\psi \mid \psi\rangle}=\frac{\operatorname{tr}\left(H e^{-\beta H}\right)}{\operatorname{tr}\left(e^{-\beta H}\right)} .
$$

By definition, $\beta=+\infty$ for the ground state and $\beta=-\infty$ for the highest excited state. In practice, the range of available $\beta$ values on a finite-size system is much smaller. With $L=21$, for instance, the first excited state has $\beta \approx 4.0$, and the second-to-highest excited state has $\beta \approx$ -0.6 [as determined from Eq. (31)]. It follows that eigenstates outside the range $4.0 \gtrsim \beta \gtrsim-0.6$ will not appear fully thermal because of the large thermal correlation length expected at low temperatures. (This can be seen, for instance, in Fig. 3, where the finite-size corrections to the linear scaling of the entanglement entropy become more prominent as temperature decreases.) Another thing to consider is that the infinite temperature eigenstate $|\psi\rangle_{\beta=0}$ is completely random and contains no information about the Hamiltonian. In a finite-size system, states near infinite temperature will also contain little information about the 


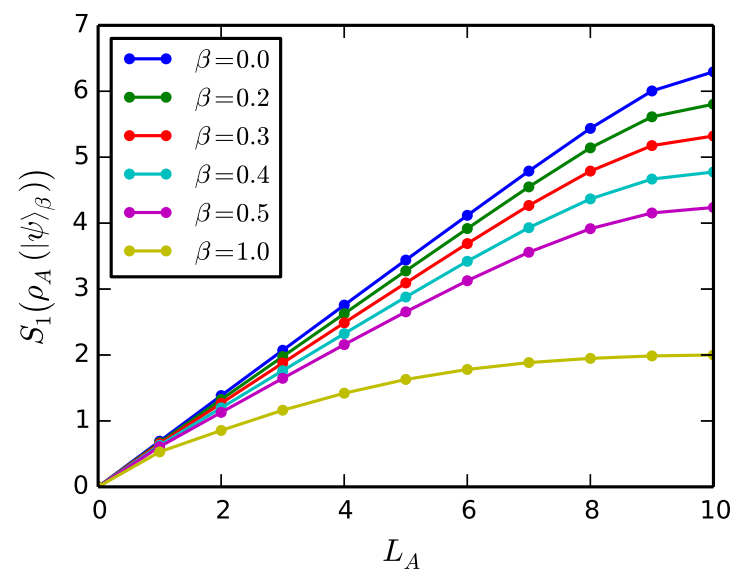

FIG. 3. Scaling of the von Neumann entanglement entropy $S_{1}$ with subsystem size for the $L=20$ system given in Eq. (30). Up to $\beta=0.5$, the scaling is linear for small $L_{A}$, suggesting that the states obey a volume law and are thus likely to satisfy ETH. The $\beta=1.0$ eigenstate, on the other hand, is clearly not linear, and it is too close to the ground state at this system size to exhibit ETH.

Hamiltonian and will therefore be unable to predict properties of the system at other energy densities. As a result of these finite-size considerations, we typically study values of $\beta$ between 0.2 and 0.5 in the remainder of this paper.

\section{VON NEUMANN AND RENYI ENTROPY OF EIGENSTATES AT FINITE TEMPERATURE}

\section{A. ETH prediction for von Neumann and Renyi entropies}

Let us consider the Renyi entropy $S_{\alpha}=$ $-[1 /(\alpha-1)] \log \left(\operatorname{tr} \rho_{A}^{\alpha}\left(|\psi\rangle_{\beta}\right)\right)$ corresponding to an eigenstate $|\psi\rangle_{\beta}$ at inverse temperature $\beta$. Assuming that ETH, as encoded in Eq. (2a), holds, $S_{\alpha}$ may be reexpressed as

$$
S_{\alpha}=-\frac{1}{\alpha-1} \log \left(\frac{Z(A, \alpha, \beta)}{Z(1, \beta)^{\alpha}}\right),
$$

where $Z(A, \alpha, \beta)$ is the partition function of the system on an $\alpha$-sheeted Riemann surface, such that subsystem $A$ has an effective temperature $(\alpha \beta)^{-1}$, while subsystem $\bar{A}$ has an effective temperature $\beta^{-1} . Z(1, \beta)$ is the regular partition function of the system [3-5]. Therefore, keeping terms only to the leading order in the subsystem size, the above expression leads to Eq. (3) as given in the Introduction $[39,40]$,

$$
\begin{aligned}
S_{\alpha} & =-\frac{1}{\alpha-1} \log \left(\frac{e^{-\alpha \beta V_{A} f(\alpha \beta)-\alpha \beta V_{\bar{A}} f(\beta)}}{e^{-\alpha \beta V_{A} f(\beta)-\alpha \beta V_{\bar{A}} f(\beta)}}\right) \\
& =\frac{\alpha}{\alpha-1} V_{A} \beta(f(\alpha \beta)-f(\beta)),
\end{aligned}
$$

where $f$ is the free energy density. Therefore, the wave function at temperature $\beta^{-1}$ can be used to calculate the free energy at temperature $(\alpha \beta)^{-1}$. Indeed, the same result also follows using the approximate form in Eq. (2b). Taking the limit $\alpha \rightarrow 1$ leads to the conclusion that the von Neumann entanglement entropy $S_{1}$ satisfies

$$
S_{1}=V_{A} s_{\text {th }}(\beta),
$$

where $s_{\mathrm{th}}(\beta)=S_{1}\left(\rho_{A, \text { canonical }}(\beta)\right) / L_{A}$ is the thermal entropy density at temperature $\beta^{-1}$. We note that Eqs. (33) and (34) are consistent with recent work studying Renyi entropies in pure states representing thermal equilibrium $[64,65]$.

\section{B. Numerical results for von Neumann and Renyi entropies}

Figure 3 shows the scaling of the von Neumann entropy $S_{1}$ as a function of subsystem size $L_{A}$ for the eigenstates $|\psi\rangle_{\beta}$ of our model [Eq. (30)]. As discussed in Sec. II C 1, we expect Eq. (35) to hold as long as $V_{A}<V_{\bar{A}}$, in the limit $V_{A}, V_{\bar{A}} \rightarrow \infty$. This implies that in the thermodynamic limit, the function $S_{1}\left(V_{A}\right)$ is expected to form an inverted triangle shape, similar to the behavior of a random pure state [Eq. (7)]. However, in a finite total system at any noninfinite temperature, $S_{1}$ is an analytic function of the ratio $V_{A} / V$ with a negative sign for $d^{2} S_{1} / d V_{A}^{2}$, as shown in Fig. 3 (note that the sign of the curvature is fixed by the strong subadditivity of entanglement). However, even in a finite system, the volume law does hold to good accuracy when $V_{A} \lesssim V / 2$, and the finite-size scaling, discussed below, indicates that the inverted triangle shape is recovered in the thermodynamic limit.

Figure 4 shows the comparison of $S_{1}, S_{2}, S_{3}$, and $S_{4}$, calculated for each individual eigenstate for a subsystem of size $L_{A}=4$ in a $L=21$ system, with their ETH predicted canonical counterparts, Eqs. (35) and (3). We use two different canonical counterparts corresponding to Eqs. (2a) and (2b), the latter version being susceptible to boundary errors, which nevertheless are expected to vanish as $V_{A}$, $V_{\bar{A}} \rightarrow \infty$. The agreement for each entropy is remarkable. It is worth reiterating that the Renyi entropies for an eigenstate $|\psi\rangle_{\beta}$ encode the free energy densities at temperatures different from $\beta^{-1}$ [Eq. (3)], and these results provide an instance of nonlocal, nonequithermal operators that satisfy ETH. Also, note that as $\alpha$ becomes larger, finite-size effects become more pronounced, because $S_{\alpha}$ probes the system at lower temperatures $(\alpha \beta)^{-1}$.

We also studied finite-size scaling of the von Neumann entropy and Renyi entropies by keeping $L_{A}$ constant and varying the total system size. The top panel of Fig. 5 shows the deviation $\Delta S_{1} / L_{A}=S_{1}\left(|\psi\rangle_{\beta}\right) / L_{A}-s_{\text {th }}(\beta)$ for eigenstates in a range of temperatures. The difference $\Delta S_{1} / L_{A}$ seemingly goes to zero faster than any inverse power of $L$, and the plot is consistent with an exponential dependence $\Delta S_{1} / L_{A} \sim e^{-L}$ or, at the very least, a power-law decay $\Delta S_{1} / L_{A} \sim 1 / L^{x}$ with $x \gg 1$ (although we should caution that inferring the precise asymptotic finite-size scaling behavior using exact diagonalization studies is an inherently difficult task). The bottom panel shows a similar plot for the 

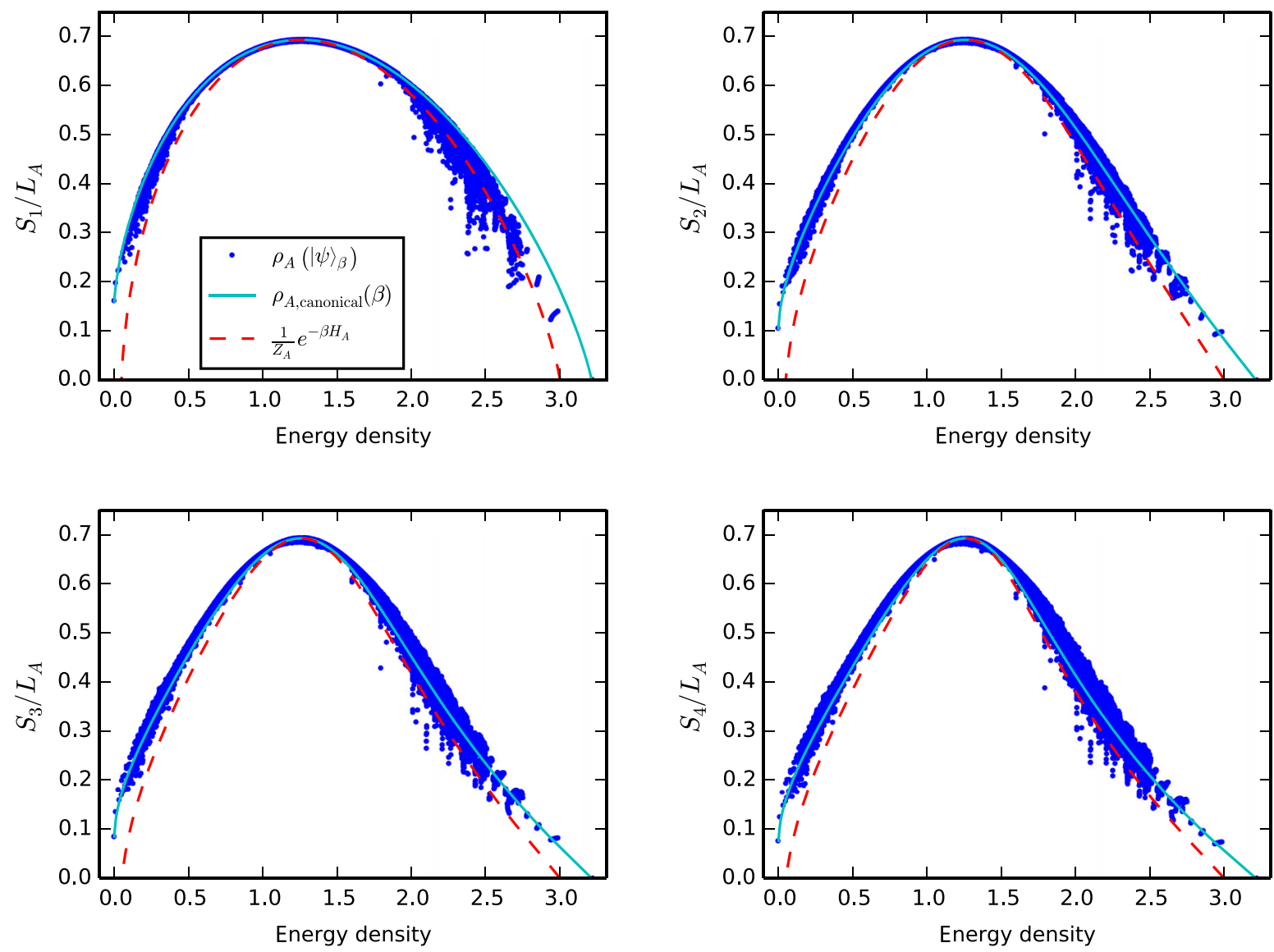

FIG. 4. The von Neumann entropy $S_{1}$ and Renyi entropies $S_{2}, S_{3}$, and $S_{4}$ for the system given in Eq. (30) with $L=21$ and $L_{A}=4$. Here, $Z_{A}=\operatorname{tr}_{A}\left(e^{-\beta H_{A}}\right)$. The entropies of the reduced density matrix at each energy density agree remarkably with the entropies calculated from the canonical ensemble, given by Eqs. (2a) and (2b).

deviation of Renyi entropy $S_{2}$ from its ETH predicted value, Eq. (3). The finite-size scaling of $\Delta S_{2}$ is relatively difficult, because unlike $S_{1}, S_{2}$ shows oscillations as a function of $L_{A}$ (see, e.g., Refs. [40,66]). Despite this, $\Delta S_{2}$ is less than a few percent of $S_{2}$ itself.

Figure 6 plots the entropy deviation $\Delta S_{1} / L_{A}$ for constant ratio $L_{A} / L$ at all available system sizes. Although it is difficult to do a detailed scaling analysis with so few points, the data strongly suggest that $\Delta S_{1} / L_{A}$ vanishes in the thermodynamic limit.

The finite-size scaling of Renyi entropies at constant ratio $L_{A} / L$ is less conclusive, as can be seen in Fig. 7. The analytical argument for the particle-number conserving model suggests that the Renyi entropies $S_{\alpha}$ for $\alpha \neq 1$ do not match their canonical counterparts when $V_{A} / V$ is held fixed. The authors of Ref. [67] arrived at similar conclusions using a different approach.

\section{EXTRACTING THE HAMILTONIAN FROM A SINGLE EIGENSTATE}

In this section, we will present numerical results that substantiate our conjecture that ETH is valid for all equithermal and nonequithermal operators when $V_{A} \ll V$ as $V \rightarrow \infty$. While our numerical results consider the case where $V_{A}$ is held constant as $V \rightarrow \infty$, we expect that all results in this section also hold true when the limits are taken such that $f \equiv V_{A} / V \rightarrow 0$ as $V_{A}, V \rightarrow \infty$. We focus here on ETH in the sense of Eqs. (2a) and (2b), as the canonical ensemble is known to be equivalent to a microcanonical ensemble when $V_{A} \ll V$. Focusing on the canonical ensemble also allows us to avoid introducing the energy width $\Delta E$ as an additional parameter in our systematic comparisons. In Sec. VII, we will explore more carefully the case when $f<\frac{1}{2}$ is finite, using both the canonical and microcanonical ensembles for comparison.

We begin by probing in detail the entanglement spectra of individual eigenstates as well as the corresponding Schmidt states. Specifically, we compare five different quantities, as shown in Fig. 8, that test the validity of Eqs. (2a)-(2c) for the model described in Sec. IV. The agreement of the spectrum of $[-1 / \beta] \log \left[\rho_{A}\left(|\psi\rangle_{\beta}\right)\right]$ with that of $[-1 / \beta] \log \left[\rho_{A \text {,canonical }}(\beta)\right]$, as well as with the actual Hamiltonian $H_{A}$ in region $A$, implies that, essentially, the Schmidt eigenvalues $\lambda_{i}$ satisfy $\lambda_{i} \propto e^{-\beta E_{A, i}}$, where $E_{A, i}$ are the eigenvalues of $H_{A}$. Similarly, the agreement with the expectation value $\left\langle u_{i}\left|H_{A}\right| u_{i}\right\rangle$ shows that the Schmidt eigenvectors 

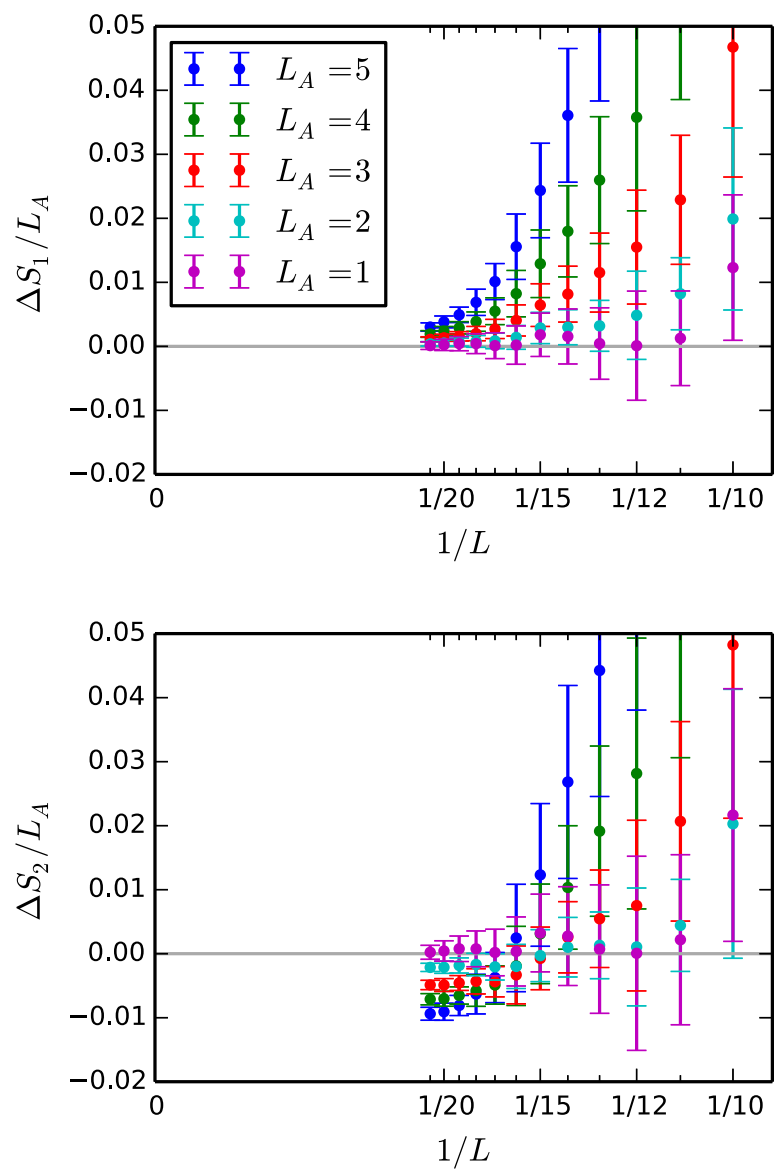

FIG. 5. Scaling of the entropy deviation $\Delta S_{\alpha} \equiv$ $S_{\alpha}\left(\rho_{A, \text { canonical }}(\beta)\right)-S_{\alpha}\left(\rho_{A}\left(|\psi\rangle_{\beta}\right)\right)$ with $1 / L$, for constant $L_{A}$, averaged over all eigenstates in the range $0.28<\beta<0.32$, for $S_{1}$ (top panel) and $S_{2}$ (bottom panel). The error bars represent one standard deviation away from the mean. For $S_{1}$, this deviation is strictly non-negative, but, for higher Renyi entropies, it can oscillate and become negative before tending to zero as $L \rightarrow \infty$.

$\left|u_{i}\right\rangle$ have the same character as the eigenvectors of the canonical reduced density matrix. It is important to emphasize that this agreement holds throughout the spectrum: The agreement near the energy density of the eigenstate itself (denoted by the dashed grey line) suggests that ETH holds for equithermal operators, while the agreement away from this energy density suggests that Eq. (1) holds for nonequithermal operators as well. (There is a slight disagreement in the spectrum near the critical energy density $e^{*}$, and we explore this in the following section.)

To probe the Schmidt eigenvectors further, we directly calculated the overlaps between the eigenvectors of the reduced density matrix $\rho_{A}\left(|\psi\rangle_{\beta}\right)$ and the eigenvectors of the thermal density matrix $\rho_{A \text {,canonical }}(\beta)$ (see Fig. 9). Again, we find excellent agreement. Again, the agreement throughout the spectrum suggests that Eq. (1) is valid for both equithermal and nonequithermal operators.

To quantify the extent to which Eq. (2a) is valid, we calculate the trace norm distance $\| \rho_{A}\left(|\psi\rangle_{\beta}\right)-\rho_{A \text {,canonical }}(\beta) \|_{1}$

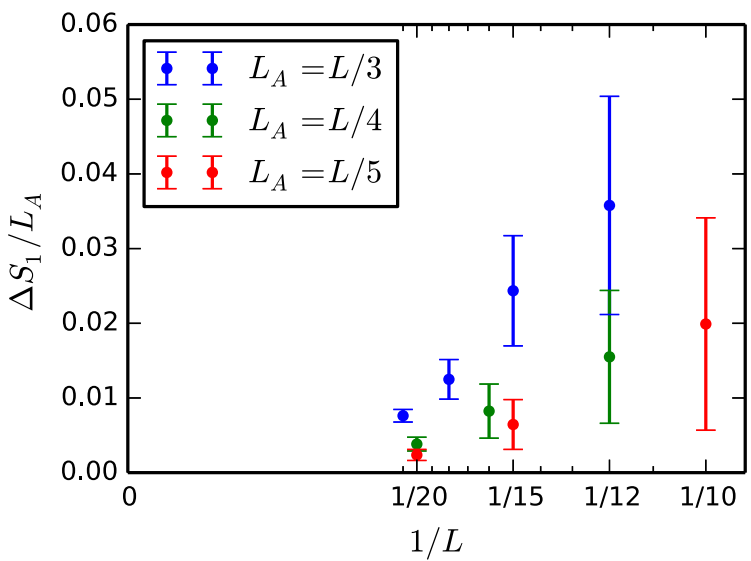

FIG. 6. Scaling of the von Neumann entropy deviation $\Delta S_{1}$ with $1 / L$, for constant ratio $L_{A} / L$, averaged over all eigenstates in the range $0.28<\beta<0.32$. As in Fig. 5, the error bars represent one standard deviation away from the mean. Even though this plot considers the case where the subsystem size $L_{A}$ becomes infinite as $L \rightarrow \infty$, the entropy deviations are going to zero rapidly as $L$ becomes larger.

between the reduced and canonical density matrices at various system sizes. The trace norm distance, defined as

$$
\begin{aligned}
& \| \rho_{A}\left(|\psi\rangle_{\beta}\right)-\rho_{A, \text { canonical }}(\beta) \|_{1} \\
& \quad \equiv \frac{1}{2} \operatorname{tr}\left[\sqrt{\left(\rho_{A}\left(|\psi\rangle_{\beta}\right)-\rho_{A, \text { canonical }}(\beta)\right)^{2}}\right],
\end{aligned}
$$

places an upper bound on the probability difference that could result from any quantum measurement on the two density matrices [68]. As such, it provides an excellent measure of how distinguishable the two density matrices are. If the trace norm distance between two finite-size density matrices is zero, they are equal to each other element by element.

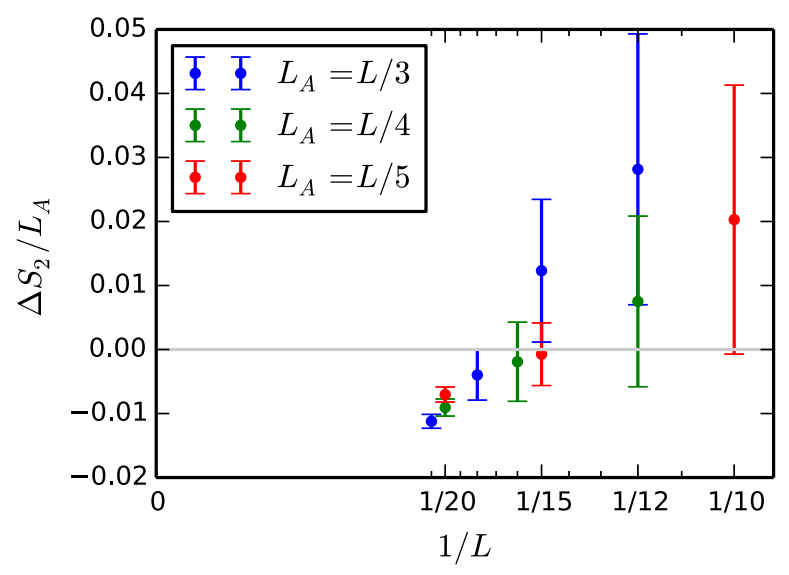

FIG. 7. Scaling of the Renyi entropy deviation $\Delta S_{2}$ with $1 / L$, for constant ratio $L_{A} / L$, averaged over all eigenstates in the range $0.28<\beta<0.32$. As in Fig. 5, the error bars represent one standard deviation away from the mean. 


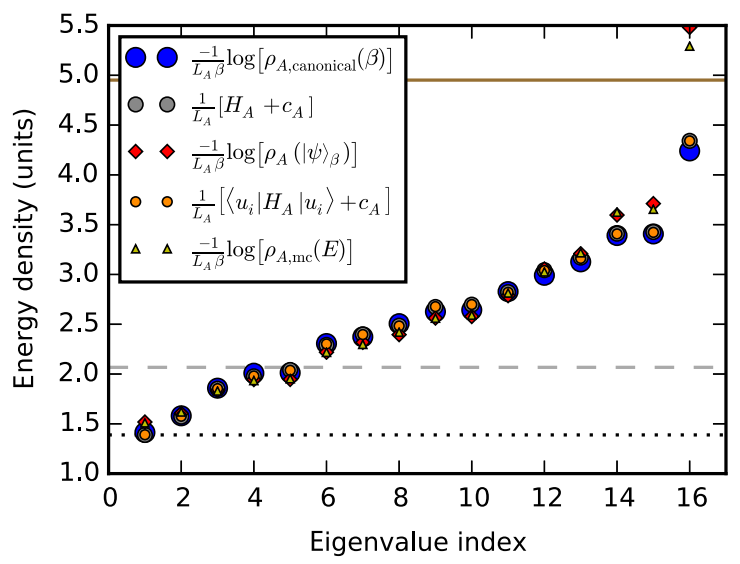

FIG. 8. Comparison of the five quantities defined in the inset for an $L_{A}=4$ subsystem at $L=21$ and $\beta=0.3$. Each quantity has been normalized so that the $y$ axis has units of energy density. The large blue circle markers show the spectrum of the canonical reduced density matrix, while the red diamond markers correspond to the eigenvalues of a reduced density matrix $\rho_{A}\left(|\psi\rangle_{\beta}\right)$ for a single eigenstate at temperature $\beta^{-1}$. The yellow triangle markers show the spectrum of the microcanonical reduced density matrix that consists of an equal-weighted mixture of the 50 eigenstates nearest in energy to the one under consideration. The grey markers show the eigenvalues of $H_{A}$ with a shift $c_{A} \equiv(1 / \beta) \log Z_{A}=(1 / \beta) \log \operatorname{tr}_{A}\left(e^{-\beta H_{A}}\right)$ so that it can be directly compared with $-(1 / \beta) \log \left[\rho_{A}\left(|\psi\rangle_{\beta}\right)\right]$ in accordance with Eq. (2b) (note, also, that the combination $H_{A}+c_{A}$ is independent of the shift of the spectrum of $H_{A}$ by an arbitrary uniform constant). Finally, the orange markers represent the expectation value of $H_{A}$, again with a shift $c_{A}$, with respect to the Schmidt eigenvector $\left|u_{i}\right\rangle$ of $\rho_{A}\left(|\psi\rangle_{\beta}\right)$. In each case, the eigenvalues/ eigenvectors are ordered from smallest to largest energy density. The horizontal lines plot the energy density $e$ (dashed grey line) and the critical energy density $e^{*}=e L / L_{A}$ (solid brown line) of the original eigenstate $|\psi\rangle_{\beta}$, with respect to the ground state energy density of $H_{A}+c_{A}$ (dotted black line).

If ETH holds for all operators in subsystem A, then the results of Ref. [16] imply that the trace norm distance should go to zero as $1 / L$. The suggestion that the trace norm distance between the pure state and thermal reduced density matrices with fixed subsystem size would tend to zero was also made in Refs. $[49,69]$. We restrict ourselves to states in a $\beta$ range given by $0.28<\beta<0.32$. In the left panel of Fig. 10, we plot the trace norm distance of every eigenstate in this $\beta$ range at $L_{A}=5$ for a few select system sizes. For each system size, the distribution of the trace norm distance is nearly constant throughout the given $\beta$ range. The right panel then takes these data for each pair of $L$ and $L_{A}$ and plots the mean and standard deviation of the trace norm distance against $1 / L$. The trace norm distance is tending toward zero at least linearly with $1 / L$, perhaps even faster.

These results, taken together, strongly support the conjecture that ETH, as given by Eq. (1), holds for all operators when $V_{A} \ll V$. The Schmidt eigenvalues and eigenvectors match at all energy densities, not just the energy density of the eigenstate. Our results also imply that when $V_{A} \ll V$, ETH as specified by Eq. (2a) holds. One consequence of this is that if $V_{A}$ is held fixed, the density matrices $\rho_{A}\left(|\psi\rangle_{\beta}\right)$ and $\rho_{A \text {,canonical }}(\beta)$ become elementwise equal in any basis as $V \rightarrow \infty$.

\section{ETH WITH FINITE RATIO $V_{A} / V$}

In this section, we consider to what extent ETH is valid when the ratio $f \equiv V_{A} / V<\frac{1}{2}$ is held fixed and finite as $V_{A}$, $V \rightarrow \infty$. When the limits are taken in this way, the canonical and microcanonical ensembles are no longer equivalent, so we consider each ensemble independently.

\section{A. Comparison with the canonical ensemble}

As demonstrated in Sec. V B, the von Neumann entropy of $\rho_{A}\left(|\psi\rangle_{\beta}\right)$ matches the thermal entropy in the thermodynamic limit even for finite $f<\frac{1}{2}$. In the current section, we consider the extent to which other quantities match between a single eigenstate and the canonical ensemble.

There is one notable equithermal operator for which Eq. (1) fails when $f$ is finite. As explained in Sec. II C 1 [see Eq. (8)], the subsystem energy variance taken from a single eigenstate is suppressed by a factor of $(1-f)$ compared with its value in the canonical ensemble. To understand this, consider first the expectation value of the operator $H_{A}^{2}-\left\langle H_{A}\right\rangle^{2}$ with respect to the canonical ensemble. This will be given by [51]

$$
\left\langle H_{A}^{2}-\left\langle H_{A}\right\rangle^{2}\right\rangle_{\rho_{A, \text { canonical }}(\beta)}=\frac{\int d\left(\delta E_{A}\right)\left(\delta E_{A}\right)^{2} e^{-\left(\delta E_{A}\right)^{2} / c V_{A}}}{\int d\left(\delta E_{A}\right) e^{-\left(\delta E_{A}\right)^{2} / c V_{A}}},
$$

where $c$ is the specific heat. Note that the probability distribution is Gaussian because it is obtained by expanding the Boltzmann factor around its maximum. On the other hand, in an eigenstate, the probability distribution will acquire an extra multiplicative factor of $e^{-\left(\delta E_{A}\right)^{2} / c V_{\bar{A}}}$ because a fluctuation $\delta E_{A}$ of energy in region $A$ is necessarily accompanied by a fluctuation $-\delta E_{A}$ in the region $\bar{A}$ since, for an eigenstate, there are no fluctuations of energy in the total system. Thus, the expectation value of $H_{A}^{2}-\left\langle H_{A}\right\rangle^{2}$ with respect to the eigenstate $|\psi\rangle_{\beta}$ is given by

$$
\begin{aligned}
& \left\langle H_{A}^{2}-\left\langle H_{A}\right\rangle^{2}\right\rangle_{\rho_{A}\left(|\psi\rangle_{\beta}\right)} \\
& =\frac{\int d\left(\delta E_{A}\right)\left(\delta E_{A}\right)^{2} e^{-\left(\delta E_{A}\right)^{2} / c V_{A}} e^{-\left(\delta E_{A}\right)^{2} / c V_{\bar{A}}}}{\int d\left(\delta E_{A}\right) e^{-\left(\delta E_{A}\right)^{2} / c V_{A}} e^{-\left(\delta E_{A}\right)^{2} / c V_{\bar{A}}}} .
\end{aligned}
$$

Equations (37) and (38) imply

$$
\frac{\left\langle H_{A}^{2}-\left\langle H_{A}\right\rangle^{2}\right\rangle_{\rho_{A}\left(|\psi\rangle_{\beta}\right)}}{\left\langle H_{A}^{2}-\left\langle H_{A}\right\rangle^{2}\right\rangle_{\rho_{A, \text { canonical }}(\beta)}}=1-V_{A} / V
$$

To demonstrate this relationship, the top panel of Fig. 11 shows scaling of the subsystem energy variance with 

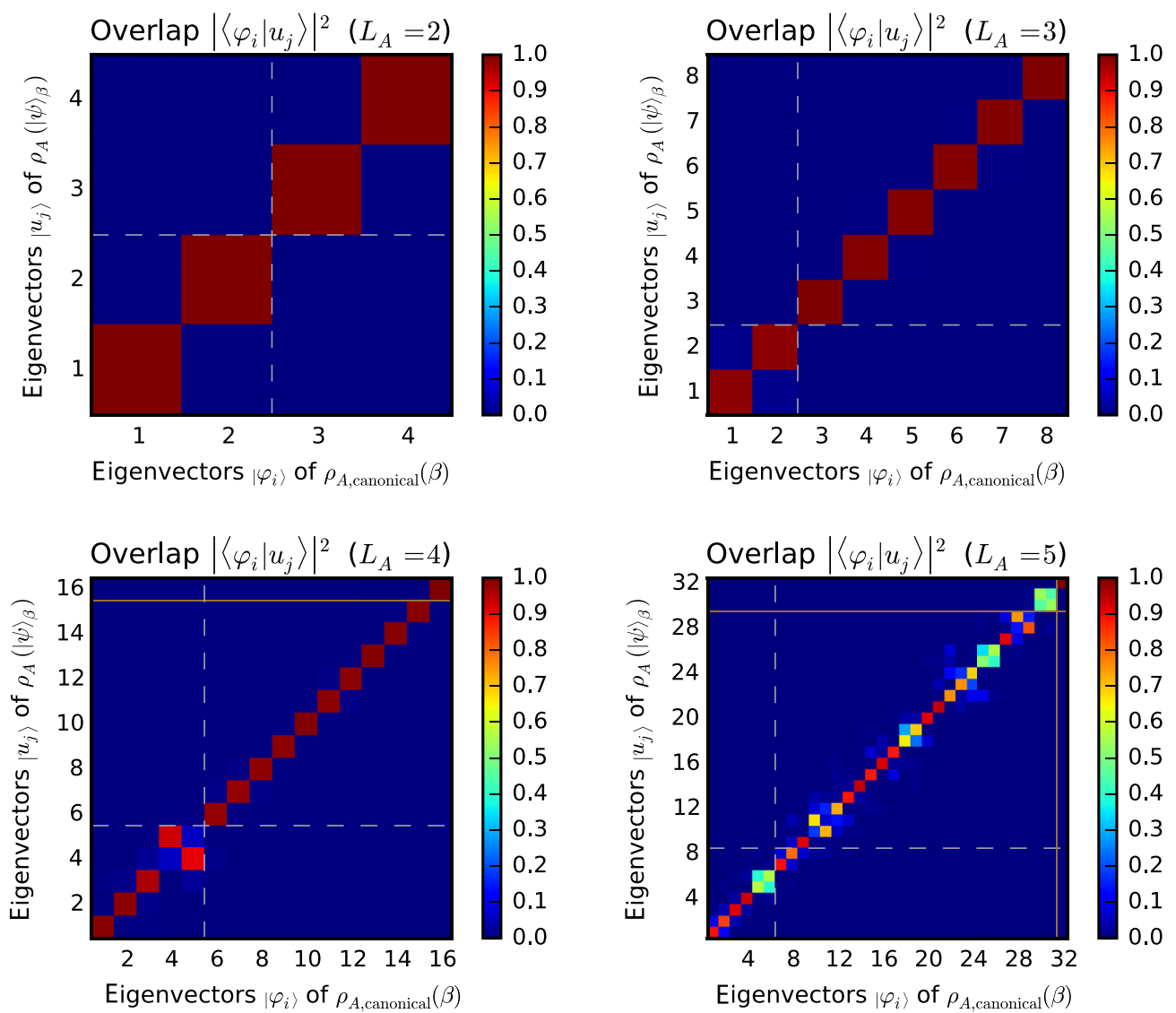

FIG. 9. Overlap between the Schmidt eigenvectors $\left|u_{j}\right\rangle$ and the eigenvectors $\left|\varphi_{i}\right\rangle$ of the canonical reduced density matrix, for an $L=21$ system with $\beta=0.3$, and subsystem sizes $L_{A}=2,3,4,5$. In each case, the eigenvectors are ordered from most significant (largest eigenvalue) to least significant (smallest eigenvalue). The locations of the energy densities $e$ (dashed grey) and $e^{*}$ (solid brown) are denoted with lines, as in Fig. 8.

subsystem size $L_{A}$ for both a single eigenstate and the canonical ensemble, for the model described in Sec. IV. While the energy variance grows linearly for $L_{A} \ll L$ in both cases, the single eigenstate energy variance has an

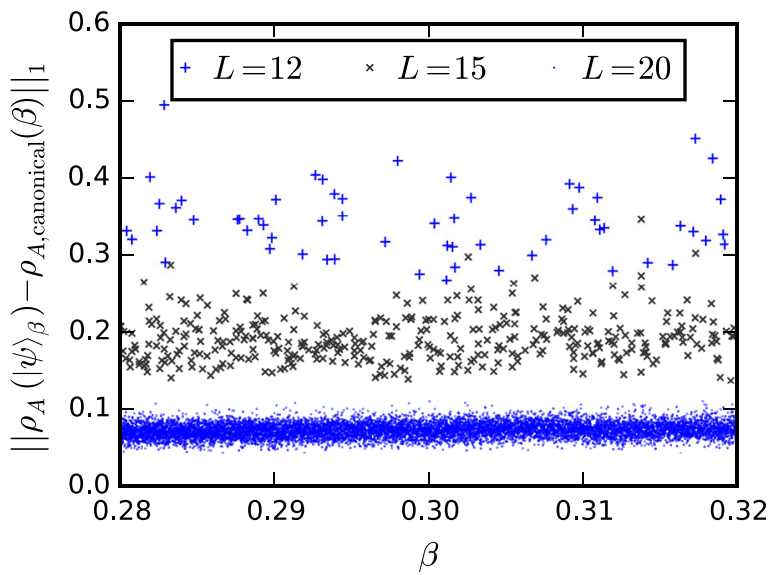

additional term that is negative and quadratic in the subsystem size, matching the prediction in Eq. (39).

The bottom panel of Fig. 11 shows, for comparison, the variance of a different operator $J_{A}$ between a single

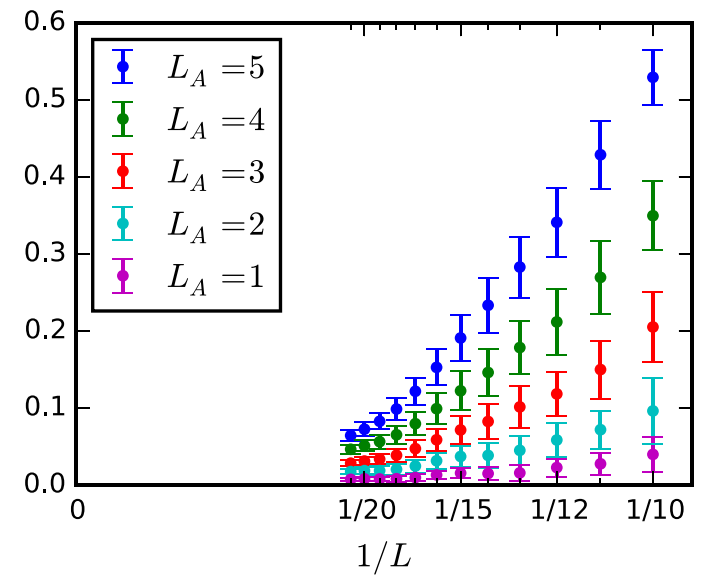

FIG. 10. Trace norm distance between the canonical density matrix $\rho_{A \text {,canonical }}(\beta)$ and the reduced density matrix $\rho_{A}\left(|\psi\rangle_{\beta}\right)$ for all eigenstates $|\psi\rangle_{\beta}$ in the range $0.28<\beta<0.32$. The left panel plots the trace norm distance for all such eigenstates with system sizes $L=12,15$, and 20, and subsystem size $L_{A}=5$. The right panel plots the mean and standard deviation of the trace norm distance in this $\beta$ range for values of $L$ up to 21 and $L_{A}$ up to 5 . 

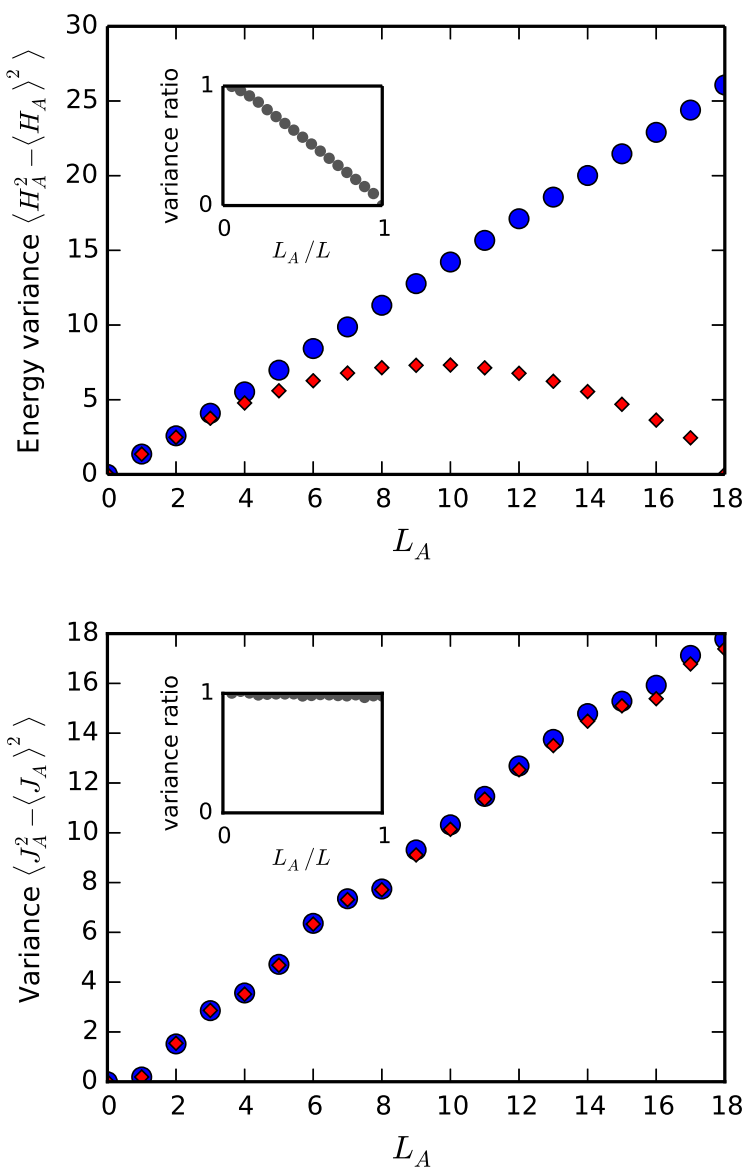

FIG. 11. Top panel: Subsystem energy variance with respect to subsystem size $L_{A}$ for both the canonical ensemble (blue circular markers) and a single eigenstate $|\psi\rangle_{\beta}$ (red diamond markers), at $L=18$ and $\beta=0.3$. The inset shows the ratio between the energy variances at each subsystem size, which is expected to fit $1-L_{A} / L$ in the thermodynamic limit [Eq. (8)]. Bottom panel: The variance of an operator $J_{A} \equiv \sum_{i=1}^{L_{A}}\left(h_{x}^{(i)} \sigma_{i}^{x}+h_{z}^{(i)} \sigma_{i}^{z}\right)+$ $\sum_{i=1}^{L_{A}-1} J_{z}^{(i)} \sigma_{i}^{z} \sigma_{i+1}^{z}$, which includes the same terms as $H_{A}$ but does not relate to energy conservation, is plotted for comparison. Here, the quantities $h_{x}^{(i)}, h_{z}^{(i)}$, and $J_{z}^{(i)}$ are each taken from the uniform distribution $[-1,1]$.

eigenstate and the canonical ensemble. The operator $J_{A}$ (defined in the figure's caption) is chosen to span the length of subsystem $A$ and to include the same terms as $H_{A}$; however, the coefficient of each term is different. The fact that the variance of $J_{A}$ matches between a single eigenstate and the canonical ensemble suggests that many equithermal operators that do not explicitly involve energy conservation will satisfy Eq. (1) at least approximately, even when $V_{A} / V$ is finite.

Let us now consider an implication of the difference in subsystem energy variance between $\rho_{A}\left(|\psi\rangle_{\beta}\right)$ and $\rho_{A, \text { canonical }}(\beta)$. This difference, which occurs only when $f$ is finite, suggests that the trace norm distance $\| \rho_{A}\left(|\psi\rangle_{\beta}\right)-$ $\rho_{A \text {,canonical }}(\beta) \|_{1}$ vanishes only when $f \rightarrow 0$. To explore this more carefully, note that the trace norm distance places a bound on the difference in expectation value of any operator $\Lambda$ that is bounded between zero and one as [68]

$$
|\operatorname{tr}(\rho \Lambda)-\operatorname{tr}(\sigma \Lambda)| \leq\|\rho-\sigma\|_{1} .
$$

In order to calculate a lower bound on trace norm distance $\| \rho_{A}\left(|\psi\rangle_{\beta}\right)-\rho_{A \text {,canonical }}(\beta) \|_{1}$ due to the variance difference, we must write the energy variance as a bounded operator that maximally differs between the two density matrices. Naively, one might be tempted to consider the operator $\mathcal{O}_{A, \beta} / \Delta^{2} \equiv\left(H_{A}-\left\langle H_{A}\right\rangle_{\beta}\right)^{2} / \Delta^{2}$, where for the operator to be bounded, $\Delta$ must be chosen to be the largest energy available to the system. Since both $\mathcal{O}_{A, \beta}$ and $\Delta$ scale linearly with $V$, the expectation value of this operator is actually zero in the thermodynamic limit for both $\rho_{A}\left(|\psi\rangle_{\beta}\right)$ and $\rho_{A \text {,canonical }}(\beta)$. Thus, no bound can be placed on the trace norm distance due to this particular operator.

Let us instead now consider a modified energy variance operator,

$$
\Lambda_{A, \beta, \Delta} \equiv P_{A, \beta, \Delta} \frac{\mathcal{O}_{A, \beta}}{\Delta^{2}} P_{A, \beta, \Delta},
$$

where $\Delta$ is an arbitrary energy scale and $P_{A, \beta, \Delta}$ projects onto the subspace where $\mathcal{O}_{A, \beta} / \Delta^{2}$ has eigenvalues in the range $[0,1]$, thus making $\Lambda_{A, \beta, \Delta}$ a bounded operator. This operator considers the energy variance within a restricted window of width $2 \Delta$ about the mean energy.

To arrive at an approximate bound due to this operator, let us assume that the energy histograms of $\rho_{A \text {,canonical }}(\beta)$ and $\rho_{A}\left(|\psi\rangle_{\beta}\right)$ are given by normal distributions with variance $\sigma_{\text {canonical }}^{2}$ and $\sigma_{\psi}^{2}=(1-f) \sigma_{\text {canonical }}^{2}$, respectively. Since both distributions have the same mean, the difference in expectation values is expected to be

$$
\begin{aligned}
D & \equiv \operatorname{tr}\left[\rho_{A, \text { canonical }}(\beta) \Lambda_{A, \beta, \Delta}\right]-\operatorname{tr}\left[\rho_{A}\left(|\psi\rangle_{\beta}\right) \Lambda_{A, \beta, \Delta}\right] \\
& =\frac{1}{\Delta^{2}} \int_{-\Delta}^{\Delta}\left[\frac{e^{-E^{2} / 2 \sigma_{\text {canonical }}^{2}}}{\sigma_{\text {canonical }} \sqrt{2 \pi}}-\frac{e^{-E^{2} / 2 \sigma_{\psi}^{2}}}{\sigma_{\psi} \sqrt{2 \pi}}\right] E^{2} d E .
\end{aligned}
$$

Given $\sigma_{\text {canonical }}$ and $f$, it is possible to find $\Delta$ numerically such that $D$ is maximized. Although $\Delta$ is proportional to $\sqrt{V}$, the value of $D$ itself is independent of $V$ as $V \rightarrow \infty$, since $\sigma_{\text {canonical }}$ also scales with $\sqrt{V}$. The maximum quantity $D$ then provides a lower bound on the trace norm distance between $\rho_{A \text {,canonical }}(\beta)$ and $\rho_{A}\left(|\psi\rangle_{\beta}\right)$ in the thermodynamic limit [70].

Let us now turn to our results on the scaling of trace norm distance with system size when the ratio $f=L_{A} / L$ is held fixed as $L, L_{A} \rightarrow \infty$, which are shown in the left panel of Fig. 12. Although there are few points available for each 

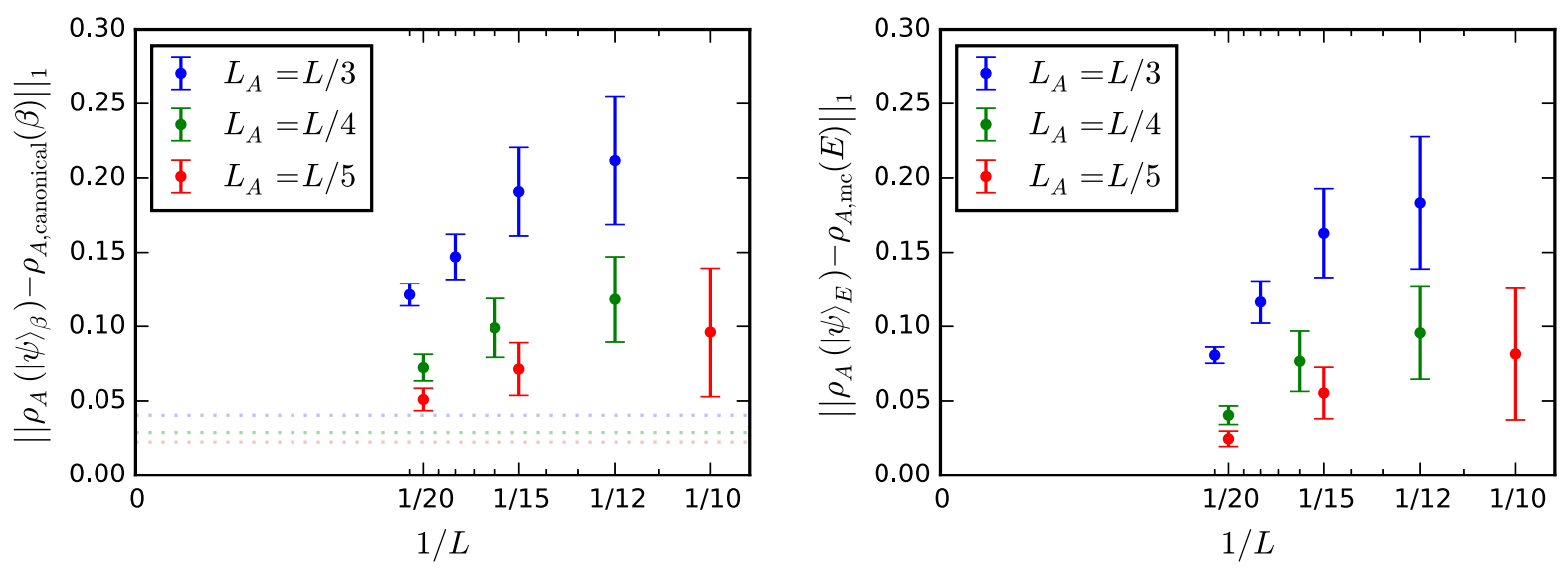

FIG. 12. Left panel: Trace norm distance between the reduced density matrices as a result of a single eigenstate and the canonical ensemble for constant ratio $L_{A} / L$ and $0.28<\beta<0.32$. As in Fig. 10, the error bars represent one standard deviation away from the mean. The horizontal lines indicate the approximate theoretical minimum each trace norm distance can take, based on the suppressed energy variance given by maximizing Eq. (42). Right panel: Trace norm distance between the reduced density matrices from a single eigenstate and a microcanonical ensemble for constant ratio $L_{A} / L$, considering all eigenstates in the range $0.28<\beta<0.32$. As in Fig. 8, the microcanonical ensemble is defined as the equal mixture of 50 eigenstates nearest in energy to the one under consideration.

ratio, the trend is clearly for the trace norm distance to decrease as $L$ increases. The horizontal dotted lines denote the theoretical minimum each trace norm distance can take, given by Eq. (42). Remarkably, for each subsystem ratio, the trace norm distance rapidly approaches this lower bound, suggesting that the bound may actually provide the result in the thermodynamic limit.

\section{B. Comparison with a microcanonical ensemble}

Now we consider to what extent the reduced density matrix from a single eigenstate equals that of a microcanonical ensemble when the subsystem ratio $f<\frac{1}{2}$ is nonzero [i.e., the potential validity of Eq. (2c)]. We first note that, unlike in the case of the canonical ensemble, the subsystem energy variance from a single eigenstate matches that from a microcanonical ensemble with vanishing energy width, independent of system size. In particular, the subsystem energy variance provides an example of an operator for which ETH in the sense of Eq. (4) holds but for which ensemble equivalence breaks down. To further investigate the potential validity of Eq. (2c), we consider the trace norm distance scaling in the right panel of Fig. 12. Unlike in the case of the canonical ensemble, we know of no examples of operators that span less than half the system for which the comparison is known to fail, and so there is no lower bound plotted in the figure. Indeed, the values are uniformly closer to zero than the comparison with the canonical ensemble, which provides strong evidence that a microcanonical ensemble is the appropriate one to compare a single eigenstate against, in agreement with Sec. II and Refs. $[49,50]$. The trace norm distance appears to approach zero at least linearly with $1 / L$, which suggests the possibility that ETH in the sense of Eq. (4) holds for all equithermal operators that span less than half the system.

It is important to emphasize that, even if the trace norm distance $\| \rho_{A}\left(|\psi\rangle_{E}\right)-\rho_{A, \mathrm{mc}}(E) \|_{1}$ indeed goes to zero in the thermodynamic limit with $V_{A} / V$ fixed, this only implies that all equithermal operators satisfy Eq. (4). When $V_{A} / V$ is fixed, nonequithermal operators will have a vanishing contribution to the trace norm distance because they probe thermodynamically rare states. One way to investigate whether Eq. (4) holds even for nonequithermal operators for a nonzero subsystem fraction is to examine the entanglement spectra.

\section{Entanglement spectrum}

We now turn to results on the entanglement spectrum when $f$ is a significant fraction of the total system size. As discussed in Sec. II C 2, if the constraint in Eq. (20) is violated, the entanglement spectrum of a single eigenstate cannot match that of the canonical ensemble above a critical energy density $e^{*}=e / f$ [see Eq. (19)], where $e$ is the energy density of the state $|\psi\rangle_{\beta}$. Figure 13 shows the comparison of spectra of five different quantities considered in Sec. VI for several different energy densities of the reference state $|\psi\rangle_{\beta}$ with $f=1 / 3$, at four different values of $\beta$. With $f=1 / 3$, the energy constraint Eq. (20) is violated, and, therefore, we expect that the entanglement spectrum from a single eigenstate should deviate from the actual spectrum of the Hamiltonian at least beyond the critical energy density $e^{*}=e / f$. We find for each value of $\beta$ that significant deviation starts to occur essentially right at this critical energy density.

While the entanglement spectrum from a single eigenstate clearly differs from the actual spectrum of the Hamiltonian and that of the canonical ensemble, it follows 

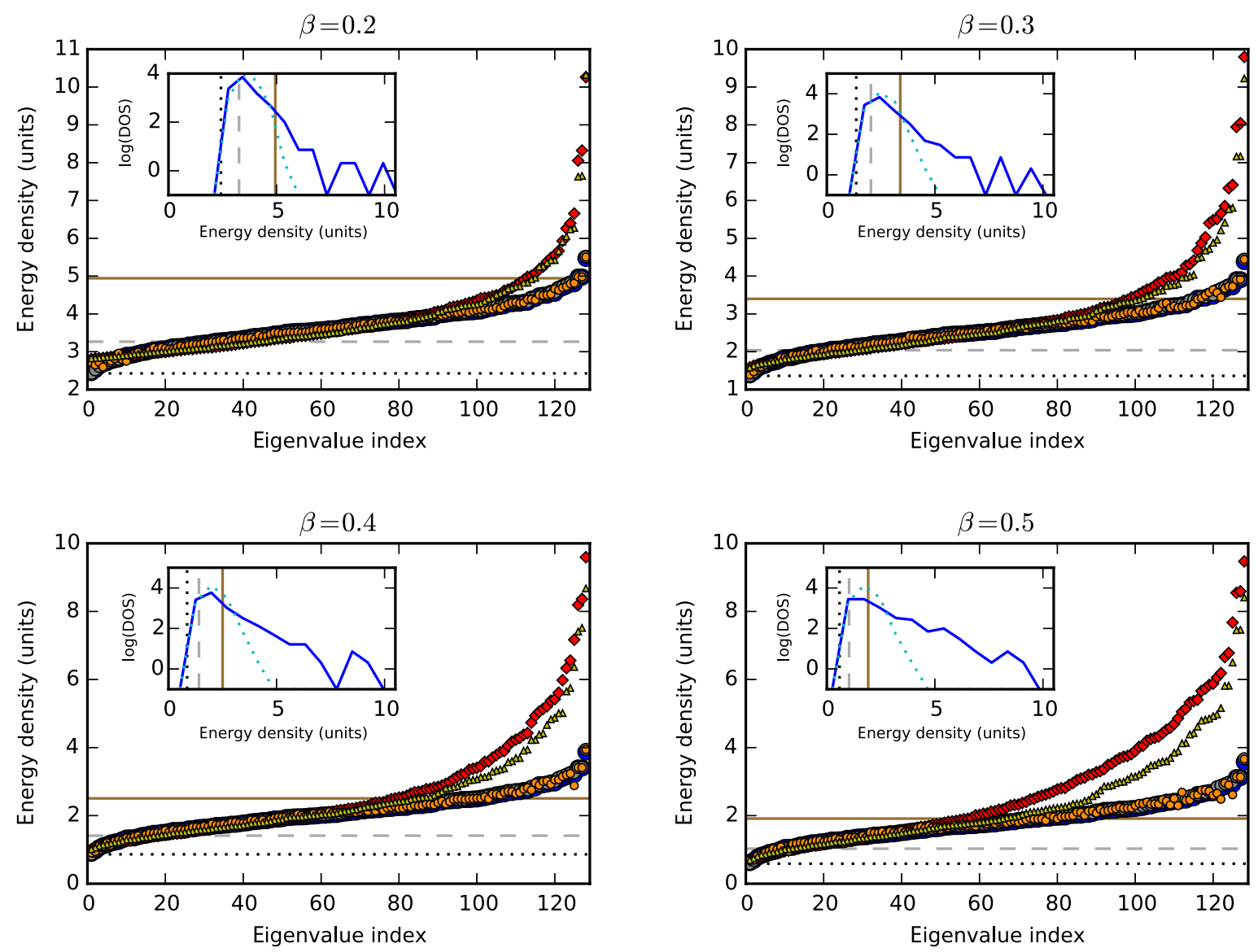

FIG. 13. Comparison of the five quantities defined in the inset of Fig. 8 for eigenstates of an $L=21$ system, with $L_{A}=7$, at $\beta=0.2$, $0.3,0.4$, and 0.5 . Each inset plots a 12-bin histogram of the log of the density of states versus the energy density: the solid blue curve from a single eigenstate $\rho_{A}\left(|\psi\rangle_{\beta}\right)$ and the dotted cyan curve from the canonical ensemble $\rho_{A \text {,canonical }}(\beta)$. We notice that, in each of the four plots, the eigenvalues of the reduced density matrix corresponding to a single eigenstate (red diamond markers) begin to deviate significantly from the eigenvalues of the canonical reduced density matrix (blue circle markers) as the energy density reaches the critical value $e^{*}$ (solid brown line), indicating the breakdown of Eq. (2a) beyond $e^{*}$.

closely the spectrum due to a microcanonical ensemble. In fact, the deviation between these two spectra increases for lower temperatures (higher $\beta$ ), where finite-size effects are expected to be the largest. These results thus leave open the possibility that the entanglement spectrum matches exactly in the thermodynamic limit when $V_{A}, V \rightarrow \infty$ such that $V_{A} / V$ is held constant.

Surprisingly, even though the entanglement spectrum from a single eigenstate does not match the actual spectrum of the Hamiltonian beyond the energy density $e^{*}$, the expectation values $\left\langle u_{i}\left|H_{A}\right| u_{i}\right\rangle / L_{A}$ continue to match the energy eigenvalues of the actual Hamiltonian. This suggests that the eigenvectors of $\rho_{A}\left(|\psi\rangle_{\beta}\right)$ match those of $\rho_{A \text {,canonical }}(\beta)$ throughout the spectrum, even though the eigenvalues do not agree everywhere. (This is also consistent with the observation that the eigenvector overlaps match even beyond $e^{*}$ in Fig. 9.) To understand this phenomenon better, we analyze the different terms in Eq. (17). As argued in Sec. IIC 2, the only way $\left\langle u_{i}\left|H_{A}\right| u_{i}\right\rangle$ can exceed the total energy $E$ of the eigenstate is if the $E_{\text {boundary }}$ term,
$E_{\text {boundary }} \equiv \sum_{j} \sqrt{\frac{\lambda_{j}}{\lambda_{i 0}}}\left\langle u_{i 0}\left|\otimes\left\langle v_{i 0}\left|H_{A \bar{A}}\right| u_{j}\right\rangle \otimes\right| v_{j}\right\rangle$,

scales with the total system size. We find that this is indeed the case, as shown in Fig. 14. In agreement with the general considerations in Sec. II C2, the Schmidt eigenvalues deviate from their canonical ETH predicted values beyond $e^{*}$ (Fig. 13) and become considerably smaller.

\section{Summary}

In this section, we compared the reduced density matrix from a single eigenstate to that of both the canonical ensemble and a microcanonical ensemble when $V_{A} / V$ is fixed and nonzero as the thermodynamic limit is taken. We find evidence that a microcanonical ensemble agrees well with a single eigenstate, and our results are consistent with the possibility that their reduced density matrices match precisely in the thermodynamic 

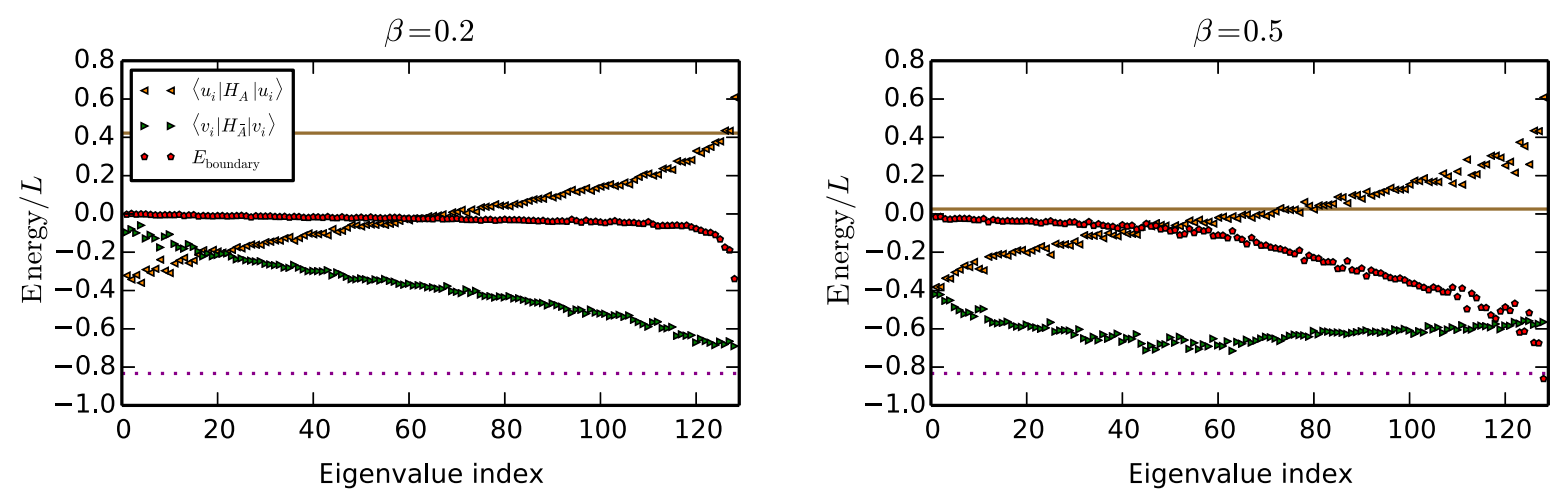

FIG. 14. Decomposition of the energy density corresponding to an eigenstate amongst the three terms in Eq. (17) for $\beta=0.2$ (left panel) and $\beta=0.5$ (right panel) at $L=21$ and $L_{A}=7$. The dotted magenta line marks the ground state of $H_{\bar{A}}$. As in Fig. 8, the solid brown line denotes the critical energy density $e^{*}$ for subsystem $A$.

limit. On the other hand, we demonstrated that while comparison with the canonical ensemble works for many operators, it nonetheless fails both for some equithermal and for some nonequithermal operators.
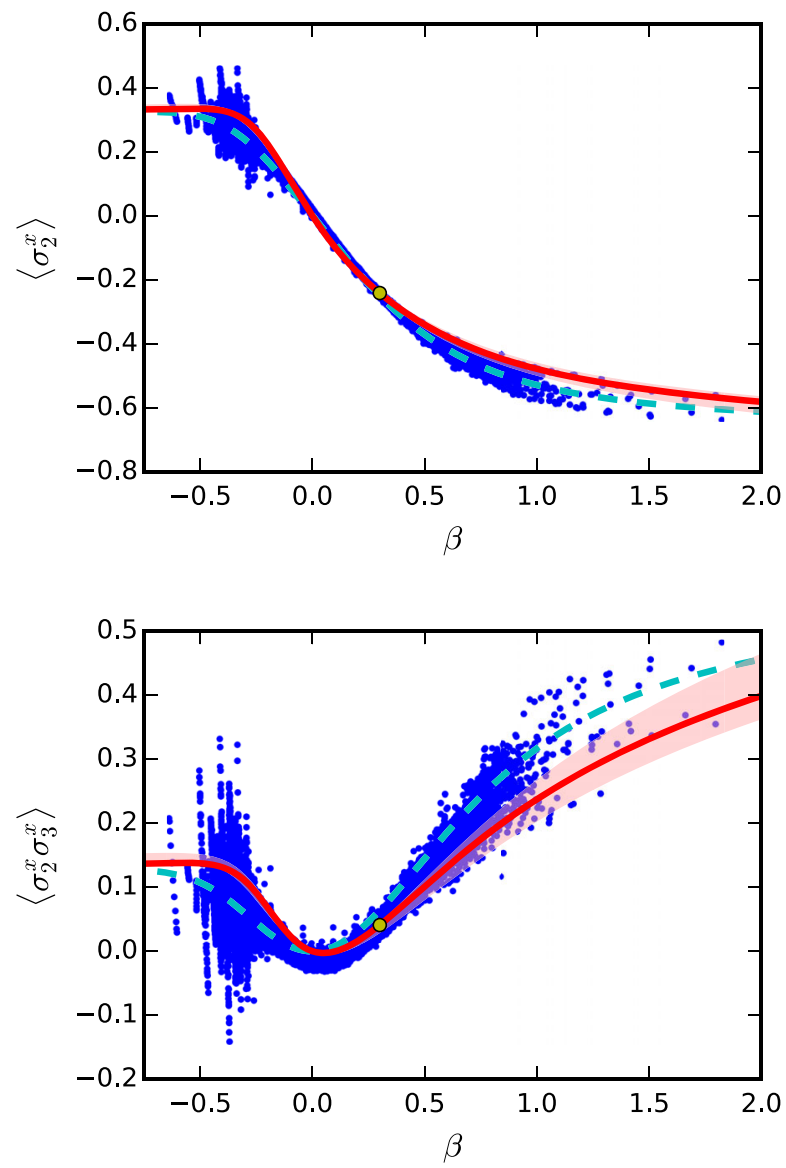

Surprisingly, while the eigenvalues between $\rho_{A}\left(|\psi\rangle_{\beta}\right)$ and $\rho_{A \text {,canonical }}(\beta)$ differ, their eigenvectors appear to match. (We leave the understanding of this result for future studies.)
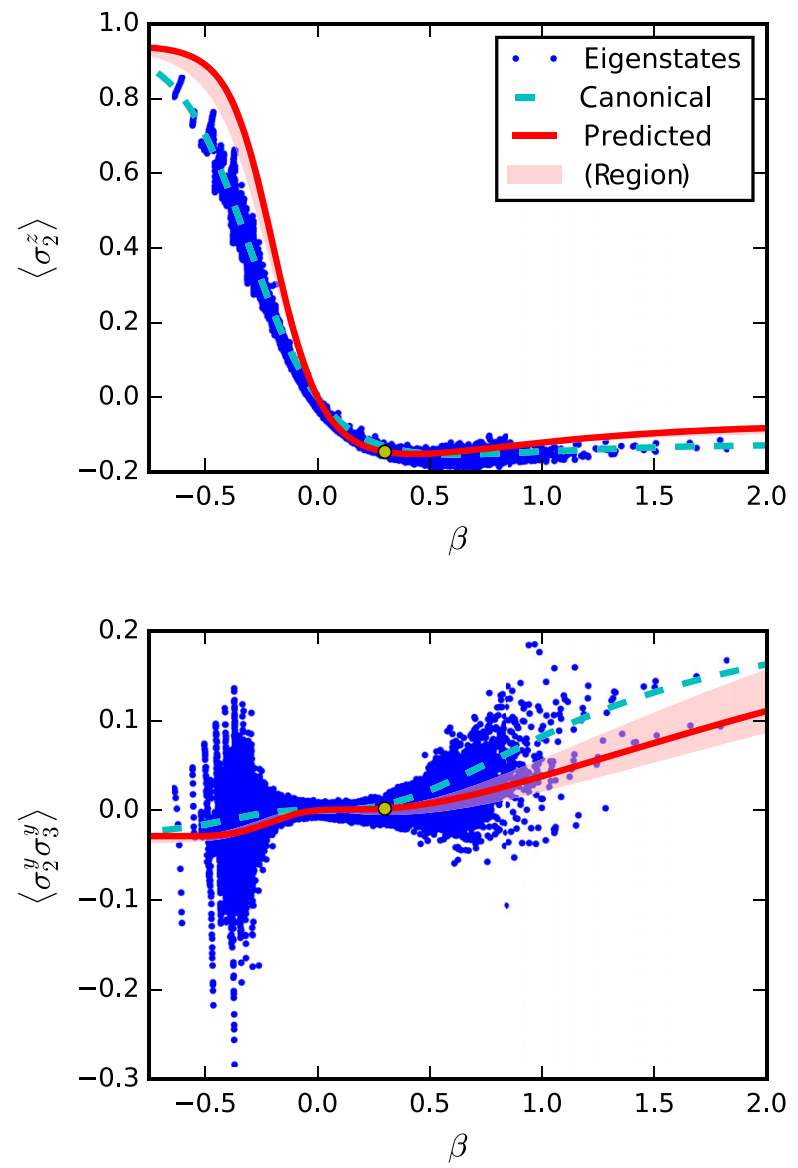

FIG. 15. Equal-time correlators for an $L=21$ system plotted against inverse temperature $\beta$. The blue dots denote the expectation value with respect to each eigenstate, the dashed cyan curve plots the expectation value in the canonical ensemble, and the red curve plots the expectation value predicted from a single eigenstate at $\beta_{0}=0.3$ (yellow dot) by raising the $L_{A}=4$ density matrix to the power $\beta / \beta_{0}$ and rescaling it to have unit trace. We also independently consider predictions from each of the 214608 eigenstates in the range $0.2<\beta_{0}<0.5$ : At each inverse temperature $\beta$, the pink shaded region contains predictions at most one standard deviation away from the mean prediction among all such eigenstates. 


\section{APPLICATIONS}

\section{A. Equal-time correlators as a function of temperature from a single eigenstate}

In the previous sections, we provided evidence that a single eigenstate encodes the full Hamiltonian. As an application of this result, we now calculate correlation functions at arbitrary temperatures using a single eigenstate $|\psi\rangle_{\beta}$. The basic idea is similar to the relation between the Renyi entropies and the free energy densities [Eq. (3)].

In particular, consider the correlation function

$$
\langle O(x) O(y)\rangle_{\beta, n}=\frac{\operatorname{tr}_{A}\left(\rho_{A}^{n}\left(|\psi\rangle_{\beta}\right) O(x) O(y)\right)}{\operatorname{tr}_{A}\left(\rho_{A}^{n}\left(|\psi\rangle_{\beta}\right)\right)},
$$

where $x, y$ are located in subsystem $A$, away from the boundary. Using Eqs. (2a) and (2b) to leading order in the subsystem size, $\langle O(x) O(y)\rangle_{\beta, n}$ equals the expectation value of the operator $O(x) O(y)$ at a temperature $(n \beta)^{-1}$.

Figure 15 shows the expectation values of local operators within subsystem $A$ as a function of $\beta$, as predicted from a single eigenstate of Eq. (30) at inverse temperature $\beta_{0}$ (indicated by a yellow dot on the red curve). We choose operators that are as far away from the subsystem boundary as possible so as to minimize the finite-size corrections. Even though the agreement with the canonical ensemble is not perfect, the qualitative trends and the numerical values match incredibly well, given the modest total system sizes to which we are restricted. These predicted correlators also undoubtedly suffer from corrections expected due to the conical singularity at the boundary of $A$ in Eq. (44). To emphasize that there is nothing special about the particular eigenstate at $\beta_{0}=0.3$, we investigate predictions from eigenstates in a broad range of energy densities, the results of which are given by the pink shaded region in Fig. 15. The shape of this region suggests that a large fraction of eigenstates are indeed able to make correct qualitative and approximate predictions at all temperatures, a remarkable feat considering the reduced density matrix (upon which all predictions are made) contains just four sites.

\section{B. Free energy density as a function of temperature from a single eigenstate}

It is also possible to predict the free energy density at all temperatures from a single eigenstate by raising the reduced density matrix to different powers $\beta / \beta_{0}$ and rescaling to have unit trace, a notion that is consistent with Eq. (3). As Fig. 16 shows, this quantity exhibits remarkable agreement with the free energy density of the system as calculated by more direct means. The prediction of free energy at all temperatures from a single eigenstate provides additional evidence that just one eigenstate contains information about the thermodynamical properties of a system at all temperatures.

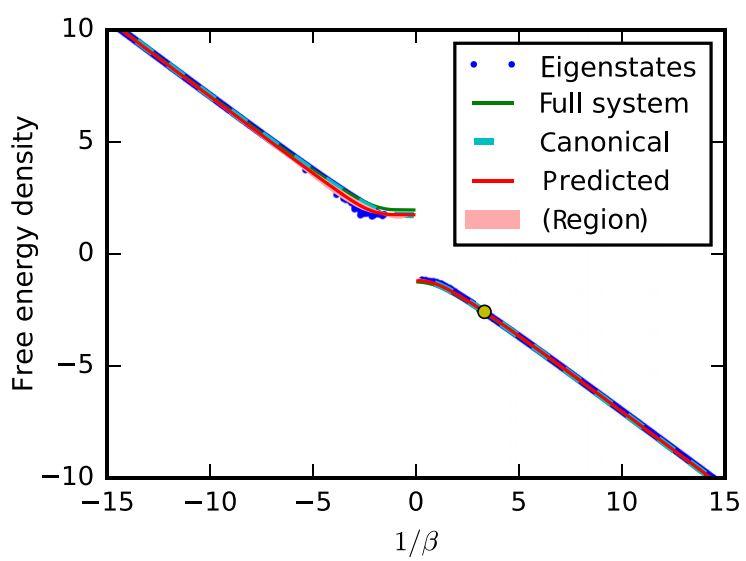

FIG. 16. Free energy density as a function of temperature for system size $L=21$. The green curve is the free energy density for the entire system in the canonical ensemble, given by $\left[\langle H\rangle-S_{\mathrm{th}} / \beta\right] / L$. The remaining curves each plot the free energy density within the $L_{A}=4$ subsystem (i.e., $\left[\left\langle H_{A}\right\rangle-S\left(\rho_{A}\right) / \beta\right] / L_{A}$ ), with respect to some reduced density matrix $\rho_{A}$ in subregion $A$ : The blue dots consider each energy eigenstate, the dashed cyan curve represents the canonical ensemble, and the red curve contains the values predicted from a single eigenstate at $\beta_{0}=$ 0.3 (itself shown in yellow). As in Fig. 15, the "predicted" density matrix is obtained by raising the eigenstate's reduced density matrix to the power $\beta / \beta_{0}$ and rescaling it to have unit trace. In fact, the eigenstate at $\beta_{0}=0.3$ is not unique: The independent predictions from each of the 214608 eigenstates in the range $0.2<$ $\beta_{0}<0.5$ all fall within the region shaded in pink.

\section{SUMMARY AND DISCUSSION}

In this paper, we analyzed the structure of reduced density matrices corresponding to the eigenstates of generic, nonintegrable quantum systems. We argued that, given an eigenstate $|\psi\rangle_{\beta}$ with energy density $e$ and a corresponding temperature $\beta^{-1}$, the reduced density matrix for a subsystem $A$ is given by

$$
\rho_{A}\left(|\psi\rangle_{\beta}\right)=\rho_{A, \text { canonical }}(\beta),
$$

where

$$
\rho_{A, \text { canonical }}(\beta)=\frac{\operatorname{tr}_{\bar{A}}\left(e^{-\beta H}\right)}{\operatorname{tr}\left(e^{-\beta H}\right)},
$$

if the condition $V_{A} \ll V$ is satisfied. This means that, for a fixed eigenstate $|\psi\rangle_{\beta}$, one can always extract the properties of the corresponding Hamiltonian at arbitrary energy densities by taking $V_{A} / V \rightarrow 0$ as the limits $V_{A}, V \rightarrow \infty$ are taken. Remarkably, even when $V_{A} / V(<1 / 2)$ is taken to be fixed and finite, one can still access many properties of the underlying Hamiltonian. However, it is important to be precise about which thermal ensemble is used for comparison because ensemble equivalence breaks down when $V_{A} / V$ is nonzero. While we constructed examples of operators for which a single eigenstate and the canonical 
ensemble disagree, our results are consistent with the possibility that ETH might hold precisely in the thermodynamic limit when $V_{A} / V<\frac{1}{2}$, as long as a microcanonical ensemble with vanishing energy window is used for comparison [Eq. (2c)].

We also introduced the notion of equithermal and nonequithermal operators. In a canonical ensemble at temperature $\beta^{-1}$, the expectation value of equithermal operators depends only on the properties of the underlying Hamiltonian at temperature $\beta^{-1}$, while the same is not true for nonequithermal operators. The validity of ETH for both equithermal and nonequithermal operators implies the ability to predict properties of a system at all temperatures given just a single eigenstate. We provided evidence that ETH is valid for both classes of operators, and, armed with this knowledge, successfully predicted local correlation functions and free energy densities at all temperatures from a single eigenstate.

We also provided analytical results for the Renyi and von Neumann entropies of infinite temperature eigenstates of a particle-number conserving model. These results substantiate our numerical results for the energy-only conserving model. In particular, we find that the von Neumann entanglement entropy for a subsystem of size $V_{A}$ equals the thermal entropy for that subsystem as long as $V_{A}<$ $V / 2$ and, therefore, follows the so-called "Page curve" [58] at all nonzero temperatures, thus generalizing the original work of Page and others [58], and in agreement with the recent work on large central charge CFTs [53-55].

Let us mention some of the practical implications of our results. Firstly, the fact that a single eigenstate encodes properties of the full Hamiltonian could potentially be a useful numerical tool. For example, one could imagine targeting a finite energy density eigenstate of a Hamiltonian $H$ by variationally minimizing the energy of the Hamiltonian $(H-E)^{2}$ with respect to trial wave functions. The techniques in this paper would then allow one to access thermal properties of the Hamiltonian without directly calculating the partition function, which could be extremely helpful for Hamiltonians that suffer from the sign problem.

Secondly, owing to the recent progress in single atom imaging techniques in cold atomic systems [71], one can now access nonlocal operators experimentally [72-75]. This potentially allows one to check some of our predictions in cold atomic systems. For example, one can perform a quantum quench on a low entanglement state, which would, at sufficiently long times, lead to a thermal state in the same sense as Eq. (2a). In principle, this allows one to determine the underlying Hamiltonian of a cold atomic system by performing tomography on a small subsystem to obtain the corresponding reduced density matrix and then taking its logarithm. Recent proposals based on Ramsey spectroscopy provide methods for directly measuring the entanglement spectrum in systems of cold atoms [76] or in nuclear spins [77]. Implementation of these protocols could allow investigation of our predictions in larger systems than would be feasible with tomography.

We conclude by posing a few questions and future directions.

In this paper, we only considered contiguous subsystems. We leave for future work a systematic study of whether Eq. (2a) continues to hold for noncontiguous subsystems when $V_{A} \ll V$.

In Sec. VIII A, we predicted equal-time correlators at all temperatures using a single eigenstate. We expect such predictions to be most successful when the thermal correlation length is short compared to the subsystem size used to take powers of the reduced density matrix. We leave for future work a study of how the accuracy of such predictions depends upon the subsystem size in comparison with the thermal correlation lengths at both the base temperature $\left(\beta_{0}^{-1}\right)$ and the target temperature $\left(\beta^{-1}\right)$.

It will also be interesting to see if a similar method works for unequal-time correlators at arbitrary temperatures. The main difference is that this requires calculating expressions such as Eq. (44) at an imaginary exponent, and estimating the effects due to the conical singularity in this case requires further study.

As mentioned above, we expect that our entire discussion carries over to time-evolved states as well, since such states are also expected to have thermal behavior at long times in the same sense as a single finite energy density eigenstate. If so, does the timescale for thermalization for a given operator (i.e., the time it takes for the expectation value of the operator to become equal to its canonical expectation value) depend on whether the operator is equithermal or nonequithermal?

Another question concerns the subleading corrections to the entanglement entropy. One expects that there always exist subleading area-law contributions to the entanglement entropy (either von Neumann or Renyi) of a single eigenstate. Are these contributions also captured correctly in the entanglement entropies calculated via a thermal reduced density matrix? Perhaps a more interesting question is whether the mutual information of two disjoint intervals (which cancels out both the volume law contribution and the area law contribution) takes the same value for a single eigenstate and its canonical counterpart.

\section{ACKNOWLEDGMENTS}

We are grateful to Leon Balents, John Cardy, Matthew Fisher, Tom Hartman, Patrick Hayden, Pavan Hosur, Max Metlitski, Olexei Motrunich, Chetan Nayak, Anatoli Polkovnikov, Marcos Rigol, Lea Santos, Brian Swingle, and especially David Huse and Xiaoliang Qi for conversations about this work. We thank anonymous referees for suggesting the calculation that led to Fig. 16 and for suggesting a comparison with a microcanonical ensemble in Sec. VII. This research was supported in part by the National Science Foundation, under Grant 
No. DMR-14-04230 (J. R. G.), by the Caltech Institute of Quantum Information and Matter, an NSF Physics Frontiers Center with support of the Gordon and Betty Moore Foundation (J. R. G.), and by the Gordon and Betty Moore Foundation's EPiQS Initiative through Grant No. GBMF4304 (T. G.). This research was also supported in part by the National Science Foundation under Grant No. NSF PHY11-25915. We also acknowledge support from the Center for Scientific Computing at the CNSI and MRL: an NSF MRSEC (DMR-1121053) and NSF CNS-0960316.

[1] J. J. Bisognano and E. H. Wichmann, On the Duality Condition for Quantum Fields, J. Math. Phys. (N.Y.) 17, 303 (1976); On the Duality Condition for a Hermitian Scalar Field, J. Math. Phys. (N.Y.) 16, 985 (1975).

[2] L. Susskind, An Introduction to Black Holes, Information and the String Theory Revolution: The Holographic Universe (World Scientific Publishing Company, Singapore, 2004).

[3] C. Holzhey, F. Larsen, and F. Wilczek, Geometric and Renormalized Entropy in Conformal Field Theory, Nucl. Phys. B424, 443 (1994).

[4] C. G. Callan and F. Wilczek, On Geometric Entropy, Phys. Lett. B 333, 55 (1994).

[5] P. Calabrese and J. Cardy, Entanglement Entropy and Quantum Field Theory, J. Stat. Mech. (2004) P06002.

[6] H. Casini, M. Huerta, and R. C. Myers, Towards a Derivation of Holographic Entanglement Entropy, J. High Energy Phys. 05 (2011) 036.

[7] M. Levin and X. G. Wen, Detecting Topological Order in a Ground State Wave Function, Phys. Rev. Lett. 96, 110405 (2006).

[8] A. Kitaev and J. Preskill, Topological Entanglement Entropy, Phys. Rev. Lett. 96, 110404 (2006).

[9] H. Li and F. D. M. Haldane, Entanglement Spectrum as a Generalization of Entanglement Entropy: Identification of Topological Order in Non-Abelian Fractional Quantum Hall Effect States, Phys. Rev. Lett. 101, 010504 (2008).

[10] Y. Zhang, T. Grover, A. Turner, M. Oshikawa, and A. Vishwanath, Quasiparticle Statistics and Braiding from Ground-State Entanglement, Phys. Rev. B 85, 235151 (2012).

[11] L. Bombelli, R. K. Koul, J. Lee, and R. D. Sorkin, Quantum Source of Entropy for Black Holes, Phys. Rev. D 34, 373 (1986).

[12] M. Srednicki, Entropy and Area, Phys. Rev. Lett. 71, 666 (1993).

[13] M. Srednicki, Chaos and Quantum Thermalization, Phys. Rev. E 50, 888 (1994); Thermal Fluctuations in Quantized Chaotic Systems, J. Phys. A 29, L75 (1996).

[14] L. D'Alessio, Y. Kafri, A. Polkovnikov, and M. Rigol, From Quantum Chaos and Eigenstate Thermalization to Statistical Mechanics and Thermodynamics, Adv. Phys. 65, 239 (2016).

[15] J. M. Deutsch, Quantum Statistical Mechanics in a Closed System, Phys. Rev. A 43, 2046 (1991).
[16] M. Srednicki, The Approach to Thermal Equilibrium in Quantized Chaotic Systems, J. Phys. A 32, 1163 (1999).

[17] B. L. Altshuler, Y. Gefen, A. Kamenev, and L. S. Levitov, Quasiparticle Lifetime in a Finite System: A Nonperturbative Approach, Phys. Rev. Lett. 78, 2803 (1997).

[18] I. V. Gornyi, A. D. Mirlin, and D. G. Polyakov, Interacting Electrons in Disordered Wires: Anderson Localization and Low-T Transport, Phys. Rev. Lett. 95, 206603 (2005).

[19] D. Basko, I. Aleiner, and B. Altshuler, Metal-Insulator Transition in a Weakly Interacting Many-Electron System with Localized Single-Particle States, Ann. Phys. (Amsterdam) 321, 1126 (2006).

[20] V. Oganesyan and D. A. Huse, Localization of Interacting Fermions at High Temperature, Phys. Rev. B 75, 155111 (2007).

[21] B. Bauer and C. Nayak, Area Laws in a Many-Body Localized State and Its Implications for Topological Order, J. Stat. Mech. (2013) P09005.

[22] J. Z. Imbrie, On Many-Body Localization for Quantum Spin Chains, J. Stat. Phys. 163, 998 (2016).

[23] R. Nandkishore and D. A. Huse, Many-Body Localization and Thermalization in Quantum Statistical Mechanics, Annu. Rev. Condens. Matter Phys. Phys. 6, 15 (2015).

[24] Y. Kagan and M. I. Klinger, Theory of Quantum Diffusion of Atoms in Crystals, J. Phys. C 7, 2791 (1974).

[25] Y. Kagan and L. A. Maksimov, Localization in a System of Interacting Particles Diffusing in a Regular Crystal, Zh. Eksp. Teor. Fiz. 87, 348 (1984) [JETP 60, 201 (1984)].

[26] T. Grover and M. P. A. Fisher, Quantum Disentangled Liquids, J. Stat. Mech. (2014) P10010.

[27] M. Schiulaz and M. Müller, Ideal Quantum Glass Transitions: Many-Body Localization without Quenched Disorder, AIP Conf. Proc. 1610, 11 (2014).

[28] M. Schiulaz, A. Silva, and M. Müller, Dynamics in Many-Body Localized Quantum Systems without Disorder, Phys. Rev. B 91, 184202 (2015).

[29] J. M. Hickey, S. Genway, and J. P. Garrahan, Signatures of Many-Body Localisation in a System without Disorder and the Relation to a Glass Transition, J. Stat. Mech. (2016) P054047.

[30] N. Y. Yao, C. R. Laumann, J. I. Cirac, M. D. Lukin, and J.E. Moore, Quasi-Many-Body Localization in Translation-Invariant Systems, Phys. Rev. Lett. 117, 240601 (2016).

[31] W. De Roeck and F. Huveneers, Asymptotic Quantum Many-Body Localization from Thermal Disorder, Commun. Math. Phys. 332, 1017 (2014).

[32] W. De Roeck and F. Huveneers, Scenario for Delocalization in Translation-Invariant Systems, Phys. Rev. B 90, 165137 (2014).

[33] W. De Roeck and F. Huveneers, Can Translation Invariant Systems Exhibit a Many-Body Localized Phase?, arXiv:1409.8054.

[34] Z. Papic, E. M. Stoudenmire, and Dmitry A. Abanin, ManyBody Localization in Disorder-Free Systems: The Importance of Finite-Size Constraints, Ann. Phys. (Amsterdam) 362, 714 (2015).

[35] M. Rigol, V. Dunjko, and M. Olshanii, Thermalization and Its Mechanism for Generic Isolated Quantum Systems, Nature (London) 452, 854 (2008). 
[36] G. Biroli, C. Kollath, and A. M. Läuchli, Effect of Rare Fluctuations on the Thermalization of Isolated Quantum Systems, Phys. Rev. Lett. 105, 250401 (2010).

[37] M. Rigol and M. Srednicki, Alternatives to Eigenstate Thermalization, Phys. Rev. Lett. 108, 110601 (2012).

[38] P. Hosur and X.-L. Qi, Characterizing Eigenstate Thermalization via Measures in the Fock Space of Operators, Phys. Rev. E 93, 042138 (2016).

[39] J. C. Baez, Renyi Entropy and Free Energy, arXiv: 1102.2098.

[40] L. Bonnes, H. Pichler, and A. M. Läuchli, Entropy Perspective on the Thermal Crossover in a Fermionic Hubbard Chain, Phys. Rev. B 88, 155103 (2013).

[41] J. M. Deutsch, Thermodynamic Entropy of a Many-Body Energy Eigenstate, New J. Phys. 12, 075021 (2010).

[42] R. R. P. Singh, M. B. Hastings, A. B. Kallin, and R. G. Melko, Finite-Temperature Critical Behavior of Mutual Information, Phys. Rev. Lett. 106, 135701 (2011).

[43] H. Tasaki, From Quantum Dynamics to the Canonical Distribution: General Picture and a Rigorous Example, Phys. Rev. Lett. 80, 1373 (1998).

[44] S. Goldstein, J. L. Lebowitz, R. Tumulka, and N. Zanghì, Canonical Typicality, Phys. Rev. Lett. 96, 050403 (2006).

[45] S. Popescu, A. J. Short, and A. Winter, Entanglement and the Foundations of Statistical Mechanics, Nat. Phys. 2, 754 (2006).

[46] L. F. Santos, A. Polkovnikov, and M. Rigol, Weak and Strong Typicality in Quantum Systems, Phys. Rev. E 86, 010102(R) (2012).

[47] M. Storms and R. R. P. Singh, Entanglement in Ground and Excited States of Gapped Free-Fermion Systems and Their Relationship with Fermi Surface and Thermodynamic Equilibrium Properties, Phys. Rev. E 89, 012125 (2014).

[48] H.-H. Lai and K. Yang, Entanglement Entropy Scaling Laws and Eigenstate Typicality in Free Fermion Systems, Phys. Rev. B 91, 081110 (2015).

[49] S. Goldstein, D. A. Huse, J. L. Lebowitz, and R. Tumulka, Thermal Equilibrium of a Macroscopic Quantum System in a Pure State, Phys. Rev. Lett. 115, 100402 (2015).

[50] S. Goldstein, D. A. Huse, J. L. Lebowitz, and R. Tumulka, Macroscopic and Microscopic Thermal Equilibrium, Ann. Phys. (Leipzig) 529, 1600301 (2017).

[51] R. K. Pathria, Statistical Mechanics (ButterworthHeinemann, Washington, DC, 1996).

[52] J. Cardy, Thermalization and Revivals after a Quantum Quench in Conformal Field Theory, Phys. Rev. Lett. 112, 220401 (2014).

[53] C. T. Asplund, A. Bernamonti, F. Galli, and T. Hartman, Holographic Entanglement Entropy from 2d CFT: Heavy States and Local Quenches, J. High Energy Phys. 02 (2015) 171.

[54] P. Caputa, J. Simon, A. Stikonas, and T. Takayanagi, Quantum Entanglement of Localized Excited States at Finite Temperature, J. High Energy Phys. 01 (2015) 102.

[55] A. L. Fitzpatrick, J. Kaplan, and M. T. Walters, Virasoro Conformal Blocks and Thermality from Classical Background Fields, J. High Energy Phys. 11 (2015) 200.
[56] E. Lubkin, Entropy of an $n$-System from Its Correlation with a k-Reservoir, J. Math. Phys. (N.Y.) 19, 1028 (1978).

[57] S. Lloyd and H. Pagels, Complexity as Thermodynamic Depth, Ann. Phys. (N.Y.) 188, 186 (1988).

[58] D. N. Page, Average Entropy of a Subsystem, Phys. Rev. Lett. 71, 1291 (1993); S. K. Foong and S. Kanno, Proof of Page's Conjecture on the Average Entropy of a Subsystem, Phys. Rev. Lett. 72, 1148 (1994); J. Sanchez-Ruiz, Simple Proof of Page's Conjecture on the Average Entropy of a Subsystem, Phys. Rev. E 52, 5653 (1995); S. Sen, Average Entropy of a Quantum Subsystem, Phys. Rev. Lett. 77, 1 (1996).

[59] We are grateful to David Huse for pointing this out to us.

[60] M. Rigol, Quantum Quenches in the Thermodynamic Limit, Phys. Rev. Lett. 112, 170601 (2014).

[61] L. F. Santos and M. Rigol, Onset of Quantum Chaos in OneDimensional Bosonic and Fermionic Systems and Its Relation to Thermalization, Phys. Rev. E 81, 036206 (2010).

[62] J. M. Deutsch, H. Li, and A. Sharma, Microscopic Origin of Thermodynamic Entropy in Isolated Systems, Phys. Rev. E 87, 042135 (2013).

[63] H. Kim, T. N. Ikeda, and D. A. Huse, Testing Whether All Eigenstates Obey the Eigenstate Thermalization Hypothesis, Phys. Rev. E 90, 052105 (2014).

[64] H. Fujita, Y. O. Nakagawa, S. Sugiura, and M. Watanabe, Universality in Volume Law Entanglement of Pure Quantum States, arXiv:1703.02993.

[65] T.-C. Lu and T. Grover, Renyi Entropy of Chaotic Eigenstates, arXiv:1709.08784.

[66] P. Calabrese, M. Campostrini, F. Essler, and B. Nienhuis, Parity Effects in the Scaling of Block Entanglement in Gapless Spin Chains, Phys. Rev. Lett. 104, 095701 (2010).

[67] A. Dymarsky, N. Lashkari, and H. Liu, Subsystem Eigenstate Thermalization Hypothesis, Phys. Rev. E 97, 012140 (2018).

[68] M. M. Wilde, Quantum Information Theory (Cambridge University Press, Cambridge, England, 2013).

[69] B. Swingle and I. H. Kim, Reconstructing Quantum States from Local Data, Phys. Rev. Lett. 113, 260501 (2014).

[70] One could also consider bounds on the trace norm distance due to higher moments $\left\langle\left(H_{A}-\left\langle H_{A}\right\rangle\right)^{n}\right\rangle$, but the most stringent bound comes from $n=2$.

[71] W. S. Bakr, J. I. Gillen, A. Peng, S. Folling, and M. Greiner, A Quantum Gas Microscope for Detecting Single Atoms in a Hubbard-Regime Optical Lattice, Nature (London) 462, 74 (2009).

[72] M. Endres et al., Observation of Correlated Particle-Hole Pairs and String Order in Low-Dimensional Mott Insulators, Science 334, 200 (2011).

[73] M. Schreiber, S. S. Hodgman, P. Bordia, H. P. Luschen, M. H. Fischer, R. Vosk, E. Altman, U. Schneider, and I. Bloch, Observation of Many-Body Localization of Interacting Fermions in a Quasirandom Optical Lattice, Science 349, 842 (2015). 
[74] A. J. Daley, H. Pichler, J. Schachenmayer, and P. Zoller, Measuring Entanglement Growth in Quench Dynamics of Bosons in an Optical Lattice, Phys. Rev. Lett. 109, 020505 (2012).

[75] R. Islam, R. Ma, P. M. Preiss, M. E. Tai, A. Lukin, M. Rispoli, and M. Greiner, Measuring Entanglement Entropy in a Quantum Many-Body System, Nature (London) 528, 77 (2015).
[76] H. Pichler, G. Zhu, A. Seif, P. Zoller, and M. Hafezi, Measurement Protocol for the Entanglement Spectrum of Cold Atoms, Phys. Rev. X 6, 041033 (2016).

[77] M. E. Beverland, J. Haah, G. Alagic, G. K. Campbell, A. M. Rey, and A. V. Gorshkov, Spectrum Estimation of Density Operators with Alkaline-Earth Atoms, Phys. Rev. Lett. 120, 025301 (2018). 